The Astrophysical JouRnAL, 562:233-253, 2001 November 20

(C) 2001. The American Astronomical Society. All rights reserved. Printed in U.S.A.

\title{
COSMIC-RAY ELECTRONS IN GROUPS AND CLUSTERS OF GALAXIES: PRIMARY AND SECONDARY POPULATIONS FROM A NUMERICAL COSMOLOGICAL SIMULATION
}

\author{
Francesco Miniati, ${ }^{1,2,3}$ T. W. Jones, ${ }^{2}$ Hyesung Kang, ${ }^{4}$ AND DongSu Ryu ${ }^{5}$ \\ Received 2001 April 20; accepted 2001 July 20
}

\begin{abstract}
We investigate the generation and distribution of high-energy electrons in the cosmic structure environment and their observational consequences by carrying out the first cosmological simulation that includes directly cosmic-ray (CR) particles. Starting from cosmological initial conditions, in addition to the gas and dark matter related quantities, we follow the evolution of CR electrons (primary and secondary) and CR ions along with a passive magnetic field. CR ions and primary electrons are injected in accordance with the thermal leakage model and accelerated in the test-particle limit of diffusive shock acceleration at shocks associated with large-scale structure formation. Secondary electrons are continuously generated through $p-p$ inelastic collisions of the CR ions with the thermal nuclei of the intergalactic medium. The evolution of the $\mathrm{CR}$ electrons accounts for spatial transport, adiabatic expansion/compression, and losses due to Coulomb collisions, bremsstrahlung, synchrotron and inverseCompton emission. The magnetic field is seeded at shocks according to the Biermann battery model, and thereafter amplified by shear flow and gas compression. We compute the emission due to the inverseCompton scattering of the simulated primary and secondary electrons off cosmic microwave background photons and compare it with the published values of the detected radiation excesses in the hard X-ray and extreme-ultraviolet wavebands. We find that the few instances of detection of hard X-ray radiation excess could be explained in the framework of IC emission from primary electrons in clusters characterized by high accretion/merger activity. On the other hand, with the only exception of measured flux from the Coma Cluster by Bowyer, Berghoefer \& Korpela, both primary and secondary CR electrons associated with the cosmic structure formation account at most for a small fraction of the radiation excess detected in the extreme-ultraviolet waveband. Next, we calculate the synchrotron emission after normalizing the magnetic field strength so that for a Coma-like cluster the volume-averaged $\left\langle B^{2}\right\rangle^{1 / 2} \simeq 3 \mu \mathrm{G}$. Our results indicate that the synchrotron emission from the secondary CR electrons reproduces several general properties observed in radio halos. These include the recently found $P_{1.4 \mathrm{GHz}}$ versus $T_{\mathrm{X}}$ relationship, the morphology and polarization of the emitting region, and, to some extent, even the spectral index. In addition, radio synchrotron emission from primary electrons turns out to be large enough to power extended regions of radio emission, resembling radio relics observed at the outskirts of clusters. Once again we find a striking resemblance between the general properties of morphology, polarization, and spectral index of our synthetic maps and those of reported in the literature.
\end{abstract}

Subject headings: acceleration of particles - cosmic rays - galaxies: clusters: general -

large-scale structure of universe - methods: numerical - X-rays: galaxies: clusters

\section{INTRODUCTION}

The existence of extended regions populated by cosmicray (CR) electrons in at least some clusters of galaxies has been apparent since the discovery more than 30 years ago of diffuse, nonthermal radio emissions from the Perseus and Coma Clusters (Leslie \& Elsmore 1961; Willson 1970). Their importance as indicators of physical processes in the clusters and cluster galaxies has grown in recent years as the number of radio-detected clusters has increased, as reports have appeared of possible diffuse nonthermal emissions in the hard X-ray (HXR) and extreme-ultraviolet (EUV) bands (e.g., Lieu et al. 1996b; Fusco-Femiano et al. 1999), and as the evidence has mounted for a rich variety of highly ener-

\footnotetext{
${ }^{1}$ Max-Planck-Institut für Astrophysik, Karl-Schwarzschild-Strasse 1, D-85741 Garching, Germany.

${ }^{2}$ School of Physics and Astronomy, University of Minnesota, Minneapolis, MN 55455.

${ }^{3}$ RTN Fellow.

${ }^{4}$ Department of Earth Science, Pusan National University, Pusan 609735, Korea.

${ }^{5}$ Department of Astronomy and Space Science, Chungnam National University, Daejeon 305-764, Korea.
}

getic phenomena in and around clusters that seem capable of energizing the electrons (e.g., Sarazin 1999; Enßlin 1999). Early on it was recognized that the radiative lifetimes of CR electrons are almost certainly too short and their diffusion too slow for them to fill an entire cluster if they are produced by a single point source (Jaffe 1977). Still, today, the origin of cluster CR electrons is not clear, although many proposals have been made. Our purpose here is to examine through direct numerical simulations the possibility that $C R$ electrons can be explained as a by-product of the very large shocks that accompany the formation and evolution of the large-scale structure of the universe.

\subsection{Radio Observations}

The number of successful detections of diffuse radio emission from clusters of galaxies has substantially increased in recent years, and some cluster statistical properties can now be investigated. The radio sources typically feature low surface brightness and an emissivity that can be described by a steep power-law spectrum (Hanish 1982; Enßlin et al. 1998). From its spectral properties and, sometimes, polarization signatures the radio emission is interpreted as synchrotron radiation, implying the presence of $\mathrm{CR}$ electrons 
and magnetic fields (Kim et al. 1989, 1990; Giovannini et al. 1993; Deiss et al. 1997; Giovannini \& Feretti 2000). Early studies on the origin of the relativistic particles conceived of them as originating in discrete radio galaxies (Jaffe 1977; Rephaeli 1977, 1979; Valtaoja 1984) or normal galaxies. More recently, the possibility of their acceleration out of the intracluster medium (ICM) thermal electron pool has been considered (Liang 1999). Models commonly assume a continual energization of the relativistic electrons by in situ first- or second-order Fermi processes (Jaffe 1977; Roettiger, Burns, \& Locken 1993; Tribble 1993; Völk, Aharonian, \& Breitschwerdt 1996; Deiss et al. 1997; Eilek \& Wetherall 1999; Wetherall \& Eilek 1999). Alternatively, such electrons could be produced as secondary products of inelastic $p-p$ collisions of $\mathrm{CR}$ ions and the thermal intracluster nuclei (Dennison 1980; Vestrand 1982; Blasi \& Colafrancesco 1999). This idea was put forward primarily to explain the widespread distribution observed for these electrons, which is in conflict with the fact that their cooling timescale is too short for them to propagate throughout a cluster from a single or even a few discrete sources (Dennison 1980).

Depending on its observed properties, the diffuse radio emission is further classified either as a "radio halo" when its morphology is regular and typically centered on and resembling the X-ray emissivity, or as a "radio relic" when it is irregular and located at the periphery of the cluster. The former typically has a low degree of polarization $(<10 \%)$. The degree of polarization in the latter case, when measured, typically turns out to be high $(\sim 30 \%$; e.g., Feretti 1999).

Radio halos are usually found in rich clusters with high ICM temperature, $T \gtrsim 7 \mathrm{keV}$, and high X-ray luminosity, $L_{\mathrm{X}}(0.1-2.4 \mathrm{keV}) \gtrsim 5 \times 10^{44}$ ergs s$^{-1}$ (Feretti \& Giovannini 1996). Since it usually extends over a linear size of about 1 $h^{-1} \mathrm{Mpc}$, the radio emission appears to be a characteristic of the whole cluster, rather than being associated with any of the individual cluster galaxies (Willson 1970). Signatures of a merging process in these clusters are often emphasized and the absence of cooling flows cited as demonstrating the connection between radio halos and mergers (a merger would likely disrupt a cooling flow; e.g., Feretti 1999). This idea seems attractive, because a merger can provide enough energy and the turbulence necessary for the amplification of the magnetic field and possibly the acceleration of relativistic particles needed to power a radio halo (Tribble 1993). In addition, since mergers are transient events, they would also explain why this type of radio source has appeared to be only occasional. Recently, however, Liang et al. (2000) found a tight and steep correlation between the radio power emitted at $1.4 \mathrm{GHz}$ and the cluster temperature, suggesting that the apparent rarity of detections should be attributed to observational insensitivity to any but the rarest, most massive clusters. Those authors also claim that the radio properties of clusters with cooling flows have not been explored sufficiently as yet.

On the other hand, the clusters hosting radio relic sources are somewhat less massive and cooler than those related to radio halos. Radio relics show no apparent correlation with merger events (although the two can be coincident; cf. Feretti 1999) and they are definitely observed in clusters containing cooling flows (Bagchi, Pislar, \& Lima Neto 1998). Radio relics are found both near the cores of clusters and at their outskirts. The spectra are typically steep, but explicit cutoffs are relatively rare even though the cooling time of the relativistic electrons is much shorter than the age of the cluster. Thus, an efficient production mechanism for an extended particle population is required in order to explain the origin of those relativistic electrons as well. In Enßlin's model (Enßlin 1999) a seed population of relativistic electrons is provided by radio ghosts, i.e., relic relativistic plasma previously injected by radio galaxies. Such a plasma would be reenergized by the encounter with a shock due to either a merger event or supersonic inflow along cosmic filaments (Enßlin et al. 1998). Given the high sound speed inside the relic plasma, such flows would actually become subsonic and propagate as a sound wave through the relic, mostly producing only an adiabatic compression of it (Enßlin \& Gopal-Krisna 2001).

\subsection{Hard X-Ray and Extreme-Ultraviolet Observations}

An energy excess with respect to the thermal emission has been detected in the HXR band of the spectrum at least in three instances: for the Coma Cluster (Fusco-Femiano et al. 1999), for A2199 (Kaastra et al. 1999), and for A2256 (Fusco-Femiano et al. 2000). An upper limit for a HXR nonthermal excess was recently also measured for A3667 (Fusco-Femiano et al. 2001). Although possibly originating from high-temperature shocked intracluster gas, HXRs are also expected as a consequence of inverse-Compton (IC) scattering off cosmic microwave background photons by electrons responsible for synchrotron emission. An approximate rms magnitude of the magnetic field can be inferred from combining HXR and synchrotron surface brightness estimates, under the assumption of a common population of relativistic particles generating the two observed radiations. However, the result for the Coma cluster indicates a value of $B \sim 0.16 \mu \mathrm{G}$ (Fusco-Femiano et al. 1999), which is more than an order of magnitude lower than magnetic field estimates through Faraday rotation measures, $B \sim 6 \mu \mathrm{G}$ (Feretti et al. 1995; see also Clarke, Kronberg, \& Böhringer 2001). An equipartition estimate of magnetic field and relativistic electrons results in $B \sim 0.4 \mu \mathrm{G}$ (Giovannini et al. 1993). There is no good theoretical argument for the existence of that kind of equipartition, however. Given these uncertainties, the origin of the HXRs is still open to debate. For this reason, nonthermal bremsstrahlung emission has also been proposed to explain the origin of the HXRs (Enßlin, Lieu, \& Biermann 1999; Sarazin \& Kempner 2000; Blasi 2000; Dogiel 2000). However, Petrosian (2001) argues that, because of the very low efficiency of nonthermal bremsstrahlung with respect to Coulomb losses, in the above model a large amount of energy would need to be dumped into the ICM, leading to an unacceptably large consequent equilibrium temperature.

Additional evidence for the existence of nonthermal populations of relativistic electrons may be suggested by observations of clusters in the EUV. There have been reported detections of EUV radiation from clusters in excess of what is expected from the hot, thermal X-ray emitting ICM (Lieu et al. 1996b, 1996a, 1999; Fabian 1996; Mittaz, Lieu, \& Lockman 1998; Kaastra 2000; Bonamente, Lieu, \& Mittaz 2001). This result appears, however, to be sensitive to the procedure adopted for the subtraction of the background (see, e.g., Lieu et al. 1996a; Mittaz et al. 1998; Bowyer, Berghoefer, \& Korpela 1999 for the details). In any case, one mechanism proposed for the origin of an EUV excess is IC scattering off cosmic microwave background photons of low-energy relativistic electrons with a Lorentz 
factor of $\gamma \sim 300$ (Sarazin \& Lieu 1998). As an alternative, the possibility that the EUV originates from warm gas produced at the interface between a population of cold clouds and the hot ICM has also been explored (Fabian 1996; Bonamente et al. 2001). However, observations of the FUV spectra with the Far Ultraviolet Spectroscopic Explorer (FUSE) has ruled out the existence of a substantial amount of warm gas, at least in the Coma and Virgo Clusters (Dixon et al. 2001).

In summary, there are indications of excess, "nonthermal" HXR emissions, and possibly EUV emissions, from some clusters that may signal the presence of substantial CR electron populations there. This information complements the radio data, even in its current, uncertain state, and adds to the motivations for understanding the likely sources and distributions of CR electron populations in clusters.

\subsection{Cosmic Shock Waves}

Collisionless plasma shocks are now generally recognized as efficient CR accelerators through the so-called "diffusive shock acceleration" (DSA) process (e.g., Drury 1983). Cosmic structure formation simulations have demonstrated that strong accretion shocks form several Mpc's from cluster cores and also penetrate deeply into cluster cores during the course of large-scale structure formation (Kang et al. 1994; Miniati et al. 2000). They have been suggested as possible sources of high-energy CRs (Kang et al. 1996, 1997), as well as seeds for the ICM magnetic field (Kulsrud et al. 1997; Ryu, Kang, \& Biermann 1998). Miniati et al. $(2000,2001)$ pointed out that those shocks associated with large-scale structure formation in principle have sufficient power to produce dynamically significant $\mathrm{CR}$ proton populations inside clusters, and by implication substantial populations of CR electrons. Those computations, based on cosmological simulations, also showed, however, that cluster shock structures are very complex. Thus, it is necessary to explore in some detail the physics of CR acceleration in this context before one can properly evaluate its role. In this paper we investigate by means of a numerical simulation CR electron injection and acceleration by such cosmic shock waves. In particular, we explore the possibility that the origin of the aforementioned radio emission and radiation excesses can be explained by the primary and secondary CR electrons, and we focus our attention on those quantities that are directly related to observations. These include the flux and the spectral features of the radiation emitted at various wavelengths, including the EUV, and soft and HXRs produced by IC scattering and radio synchrotron emission from the CR electrons.

There have been previous attempts to study the observational consequences of $\mathrm{CR}$ electrons with simulations (e.g., Roettiger, Burns, \& Stone 1999a; Takizawa \& Naito 2000; Dolag \& Enßlin 2000). However, for the first time we include the CR population explicitly in a fully cosmological simulation by computing particle injection, acceleration, and energy losses in accord with the properties of the local environment in which the particles are propagating, and starting from cosmological initial conditions. Here, our focus is CR "electrons"; CR "protons" (as the dominant ionic CR component) are discussed in a companion paper (Miniati et al. 2001). We note the suggestions that there are additional sources of CRs in clusters, of course, such as active galaxies (Völk et al. 1996; Enßlin et al. 1997;
Berezinsky, Blasi, \& Ptuskin 1997). We do not attempt to include them in our current work, since our goal is to understand the role of structure shocks. However, we do call attention in our discussions to some expected differences between shock CR sources and isolated sources, as appropriate. Our modeling efforts provide some initial clues as to how these different sources can be distinguished observationally.

The paper is organized as follows. In $\S 2$ we describe the numerical methods. In $\S 3$ we present the main results of our simulation, and the conclusions are given in $\S 4$.

\section{THE NUMERICAL SIMULATION}

In order to investigate the various issues related to $C R$ electrons, we have carried out a cosmological numerical simulation that follows simultaneously the evolution of dark "matter" particles, gas quantities, and magnetic field, plus CR ions, primary CR electrons injected from thermal plasma, and secondary CR electrons. The cosmological aspects of the simulation setup are presented in $\S 2.1$. For the matter part, we have used an Eulerian total variation diminishing (TVD) hydro $+N$-body cosmology code (Ryu et al. 1993). The magnetic field is seeded at shocks in accordance with the Biermann battery mechanism and is thereafter followed passively (Kulsrud et al. 1997). After being generated at shocks, the magnetic field is amplified by shear flow through stretching and by field compression. Numerical resolution in this simulation, however, is insufficient to produce field strengths of the same order of magnitude as observed in today's groups/clusters of galaxies from realistic seed fields. Therefore, the strength of the magnetic field is normalized a posteriori to be consistent with the values inferred from observations of Faraday rotation measure through clusters (e.g., Clarke et al. 2001). Finally, the evolution of the different CR populations in the simulation is computed by the code COSMOCR (Miniati 2001). In the following sections we provide a brief description of the physical processes included in this code, i.e., CR injection at shocks and spatial transport $(\S 2.2)$, production of secondary electrons $(\S 2.3)$, and energy losses $(\S 2.4)$.

\subsection{Simulation Set-up}

Since the computation involves a quantity never before simulated in this context, i.e., the CRs, we have elected to begin the study from the relatively simpler case of a standard cold dark matter (SCDM) model, based a modest resolution simulation with $256^{3}$ cells and $128^{3}$ dark matter (DM) particles. We leave the currently more favored $\mathrm{CDM}+\Lambda$ model as the natural follow-up step for future work. Although it is now well known that SCDM is not a viable model (e.g., White et al. 1993; Ostriker 1993), the key feature required of the simulation is a distribution of collapsed objects whose properties resemble those observed in the real universe, thus allowing comparisons of their general characteristics. To accomplish this within SCDM, we set the value for the rms of today's density fluctuation on a scale of $8 h^{-1} \mathrm{Mpc}$ to be $\sigma_{8}=0.6$, incompatible with $C O B E$ results and a SCDM universe, yet a value that induces the emergence of a reasonable population of groups/clusters in simulations of large-scale structure formation (Ostriker \& Cen 1996). In addition to $\sigma_{8}$, we also define the following parameters: the spectral index for the initial power spectrum of perturbations, $n=1$, the normalized Hubble con- 
stant, $h \equiv H_{0} /\left(100 \mathrm{~km} \mathrm{~s}^{-1} \mathrm{Mpc}^{-1}\right)=0.5$, the total mass density $\Omega_{M}=1$, and the baryonic mass fraction $\Omega_{b}=0.13$.

The size of our simulated region is chosen as a compromise between a simulated cosmological volume large enough to contain groups/clusters of galaxies and a resolution sufficient to capture the main structural features of the simulated objects (groups/clusters and their shocks). We have selected a cubic region of size $50 h^{-1} \mathrm{Mpc}$ at the current epoch. With $256^{3}$ cells, this corresponds to a spatial resolution of about $200 \mathrm{~h}^{-1} \mathrm{kpc}$. In general, a coarse grid limits the finest structure that can form and consequently influences properties of the simulated objects. Then, because density peaks are smoothed, estimates of any quantity depending on the square of the density, such as the thermal bremsstrahlung emission $\left(\propto n_{\text {thermal }}^{2}\right)$, will be reduced. The effect is stronger for lower temperature groups/clusters, which have structures similar to the larger clusters, but also smaller physical scales. Thus, because of resolution effects, the emissivity from secondary electrons (whose production roughly scales as $\propto n_{\text {thermal }}^{2}$ ) will be systematically underestimated and will have a steeper temperature dependence. Previous resolution studies carried out to test the performances of the code employed here indicate that the thermal emission is underestimated by a factor of a few, although the order of magnitude should be correct (Cen \& Ostriker 1999). On the other hand, the resolution errors on the emissivity from primary electrons should be less severe compared to the case of secondary electrons.

\subsection{Injection at Shocks and Spatial Transport}

Ions and electrons injected at shocks from thermal plasma should provide the main source of CRs and are usually referred to as "primary" CRs. However, the hadronic interaction of high-energy ions with the thermal nuclei of the background gas generates, among other secondary decay products, relativistic electrons and positrons. The COSMOCR code follows explicitly the injection, acceleration, transport, and energy losses of three CR species: ions, primary electrons, and secondary electrons. The complete CR computational methods are described in Miniati (2001), and the detailed results of the CR ion simulation are presented in Miniati et al. (2001).

The injection of CR ions at shocks is computed in the "test particle limit" and according to a "thermal leakage" model (Kang \& Jones 1995). The fraction of suprathermal protons injected into CRs from plasma flowing through a shock is controlled by an injection parameter, $c_{1}$, which defines the momentum threshold for injected protons as $p_{\text {inj }}=c_{1} 2\left(m_{p} k T_{\mathrm{sh}}\right)^{1 / 2}$. In this expression $m_{p}$ is the proton mass, $k_{\mathrm{B}}$ is Boltzmann's constant, and $T_{\mathrm{sh}}$ is the postshock plasma temperature. We adopt $c_{1}=2.6$, which leads to injection of $\sim 10^{-4}$ of the thermal protons passing through shocks. This injection rate is consistent with observation and theoretical studies of DSA (Lee 1982; Quest 1988; Kang \& Jones 1995; Malkov 1998).

Since thermal electrons have gyroradii much smaller than analogous ionic gyroradii, they cannot be injected in the same fashion as protons. A preacceleration process is needed before electrons can be introduced into the DSA process (e.g., McClements et al. 1997). However, the physics underlying the injection at shocks of primary electrons is very complex and not well understood. Therefore, following the approach of Ellison, Berezhko, \& Baring (2000), we compute the injection rate of $\mathrm{CR}$ electrons by assuming a fixed value $R_{e / p}$ for the ratio of primary electrons to protons at relativistic energies. There is some observational evidence that this ratio is in the range of $1 \%-5 \%$ for Galactic CRs (Mueller \& Tang 1987; Mueller et al. 1995). Thus, the normalization of the number density in our calculations is controlled through the ionic injection rate for secondary electrons and both the ionic injection rate and $R_{e / p}$ for primary electrons. For convenience, we report our results with $R_{e / p}=1$. Thus, the emissivity for the primary electrons needs to be multiplied by an additional factor of $R_{e / p} \sim$ $0.01-0.05$ in order to get practical values.

Spatial diffusion of the GeV CR electrons on the scales of interest here occurs on timescales much longer than advection and can therefore be neglected in regions dominated by smooth flows, i.e., shockless regions (Jones, Ryu, \& Engel 1999; Miniati 2001). However, the diffusive properties of the CRs are still important because: (1) the acceleration process depends explicitly on diffusive propagation immediately adjacent to the shock, and both (2) the maximum energy reachable by the accelerated particles and (3) the maximum energy of the particles confined within the ICM depend on the diffusion coefficient. As for point 1, except for extremely high energy CRs, the scales on which that diffusion applies are far too small to be modeled in a cosmological simulation, and the timescale for the acceleration is effectively instantaneous by the same measures. Therefore, as explained in Jones et al. (1999), the injected CR protons and electrons are redistributed in energy according to a power law as prescribed by DSA theory (e.g., Drury 1983). Analogously, a preexisting population of CR electrons passing through a shock is compressed, and, if appropriate, its spectrum flattened according to the Mach number of the shock. Additional details of these procedures can be found in Miniati (2001). As for points 2 and 3, the diffusion coefficient and the associated mean free path are usually computed in the pitch-angle resonant scattering approximation (e.g., Drury 1983) as

$$
\kappa_{\|}=\frac{1}{3} r_{\mathrm{L}} v \frac{B^{2} / 8 \pi}{k W_{k}}
$$

where $W_{k}$ is the magnetic energy density per wavenumber evaluated at $k \sim 1 / r_{\mathrm{L}}$, i.e., the inverse of the Larmor radius. Thus, the diffusion coefficient is inversely proportional to the level of resonant wave turbulence, and therefore depends on the spectral properties of the turbulent magnetic field. The diffusion coefficient determines the rate of energy gain which, when compared with the dominant energy loss rate, gives an estimate of the maximum energy of the accelerated particles. It is shown that in the case of an average ICM magnetic field of value $\sim 0.15 \mu \mathrm{G}$ and with a Kolmogorov power spectrum, the upper limit for the energy of the accelerated electrons could be low enough to produce a cutoff in the radio synchrotron emission around a few $\mathrm{GHz}$ (Blasi 2001). Such a cutoff, however, disappears if one makes the assumption of Bohm diffusion (Blasi 2001). In any case, with the normalization adopted in $\S 2.4$, the magnetic field in the groups/clusters of interest here (with temperature $T_{\mathrm{X}} \gtrsim 1 \mathrm{keV}$ ) is always larger than the above value of $0.15 \mu \mathrm{G}$. Therefore, such a cutoff should not affect the spectra of our simulated clusters. Finally, we point out that diffusive escape of the CR-ion component, which is responsible for the production of the secondary electrons, 
only affects particles with energy above $\sim 2 \times 10^{15} \mathrm{eV}$ (Völk et al. 1996; Berezinsky et al. 1997). This component was not included in our simulation (Miniati et al. 2001) and should not be relevant for the secondary electrons in the energy range investigated here (e.g., Mannheim \& Schlickeiser 1994).

\subsection{Production of Secondary Electrons}

The main channels for the production of secondary electrons are given by (Gaisser 1990)

$$
\begin{aligned}
& p+p \rightarrow \pi^{ \pm}+X, \\
& p+p \rightarrow K^{ \pm}+X,
\end{aligned}
$$

where $X$ indicates all the other by-products of the reactions. Subsequently, charged pions and kaons decay mainly into muons (although a fraction of kaons also produce pions), from which the secondary electrons originate (see Miniati 2001 for more details on the processes included in COSMOCR). In addition to $p+p$ inelastic collisions, the above cascades are also triggered by the interaction of $p+\mathrm{He}, \alpha+\mathrm{H}$, and $\alpha+\mathrm{He}$, which, for example, increase the overall yield of secondary $e^{-}$by a factor of 1.4 for a metal composition similar to the interstellar medium (Dermer 1986). Here we have assumed a helium number fraction of $7.3 \%$ for the background gas and a ratio $(\mathrm{H} /$ $\mathrm{He}) \simeq 15$ at fixed energy per nucleon for the CRs (Meyer, Drury, \& Ellison 1997).

In general, we write the production spectrum of secondary electrons as (Moskalenko \& Strong 1998)

$$
\begin{aligned}
i_{s}\left(\varepsilon_{s}\right)= & N_{\mathrm{H}} \sum_{i=\pi, K} \int_{\varepsilon_{p}^{\min }}^{\infty} d \varepsilon_{p} J_{p}\left(\varepsilon_{p}\right)\left\langle\zeta \sigma_{i}\left(\varepsilon_{p}\right)\right\rangle \\
& \times \int_{\varepsilon_{i}^{\min }\left(\varepsilon_{s}\right)}^{\varepsilon_{i}^{\max }\left(\varepsilon_{p}\right)} d \varepsilon_{i} F_{s}\left(\varepsilon_{s}, \varepsilon_{i}\right) F_{i}\left(\varepsilon_{i}, \varepsilon_{p}\right),
\end{aligned}
$$

where $J_{p}\left(\varepsilon_{p}\right)$ is the proton flux, $\left\langle\zeta \sigma_{i}\left(\varepsilon_{p}\right)\right\rangle$ is the inclusive cross section $^{6}$ of the processes given in equations (2) and (3), $\varepsilon_{p}^{\mathrm{min}}$ is the minimum proton energy required to produce a meson of energy $\varepsilon_{i}^{\min }, \varepsilon_{i}^{\max }$ is the maximal energy of the produced meson, $\varepsilon_{i}^{\min }$ in turn is the minimum required energy of a meson for production of secondaries of energy $\varepsilon_{s}$, and finally $F_{i}\left(\varepsilon_{\pi, K}, \varepsilon_{p}\right)$ is the spectra of $\pi$ and $K$ produced from the collision of a proton of energy $\varepsilon_{p}$, and $F_{s}\left(\varepsilon_{s}, \varepsilon_{\pi, K}\right)$ the distribution of secondaries from the subsequent decay of the above collision products. The cross sections were computed according to the GALPROP routines by Moskalenko \& Strong (1998), to which we refer the reader for a full summary of the technique employed here for the calculation of the secondary electrons.

\subsection{Radiative Losses}

COSMOCR (Miniati 2001) accounts for various energyloss mechanisms suffered by the CR electrons in different energy ranges of interest here. To do this, a Fokker-Planck equation that has been integrated over finite momentum bins is solved. This takes advantage of the near-power-law form of the CR momentum distribution. In effect, the momentum space is divided into $N_{p}=8$ logarithmically equidistant intervals, bounded by $\hat{p}_{0}, \ldots, \hat{p}_{N_{p}}=\hat{p}_{\max }$, which we refer to here as "momentum bins." The electron distribution function $f(\hat{p})$, as a function of the normalized momentum $\hat{p} \equiv p / m_{e} c$, in each spatial cell and for each

\footnotetext{
${ }^{6}$ Inclusive means that which describes the process $p+p \rightarrow i+X$, where $i$ is in general a secondary particle.
}

momentum bin is approximated by the piecewise power law

$$
f\left(x_{i}, \hat{p}\right)=f_{j}\left(x_{i}\right) \hat{p}^{-q_{j}\left(x_{i}\right)}, \quad 1<\hat{p}_{j-1} \leq \hat{p} \leq \hat{p}_{j},
$$

where $f_{j}\left(\boldsymbol{x}_{i}\right)$ and $q_{j}\left(\boldsymbol{x}_{i}\right)$ are the number normalization and logarithmic slope, respectively, for a given cell and $\hat{p}$ bin. With the above definition, the number density of particles is given by $d N=4 \pi \hat{p}^{2} f(\hat{p}) d \hat{p}$.

Within each momentum bin $j$, and at each spatial grid point $\boldsymbol{x}_{i}$, we follow the CR electrons total number density and kinetic energy density, defined as

$$
\begin{aligned}
& n\left(\boldsymbol{x}_{i}, \hat{p}_{j}\right)=4 \pi \int_{\hat{p}_{j}}^{\hat{p}_{j+1}} f\left(\boldsymbol{x}_{i}, \hat{p}\right) \hat{p}^{2} d \hat{p}, \\
& \varepsilon\left(\boldsymbol{x}_{i}, \hat{p}_{j}\right)=4 \pi \int_{\hat{p}_{j}}^{\hat{p}_{j+1}} f\left(\boldsymbol{x}_{i}, \hat{p}\right) T(\hat{p}) \hat{p}^{2} d \hat{p},
\end{aligned}
$$

where $T(\hat{p})=(\gamma-1) m_{e} c^{2}$ is the relativistic kinetic energy. Furthermore, for each momentum bin $q_{j}\left(\boldsymbol{x}_{i}\right)$ is determined self-consistently from the values of $n\left(\boldsymbol{x}_{i}, \hat{p}_{j}\right)$ and $\varepsilon\left(\boldsymbol{x}_{i}, \hat{p}_{j}\right)$ defined above (see Miniati 2001 for details). With this formalism, the evolution of $n\left(\boldsymbol{x}_{i}, \hat{p}_{j}\right)$ in momentum space is described by the equation

$$
\begin{aligned}
\frac{\partial n\left(\boldsymbol{x}_{i}, \hat{p}_{j}\right)}{\partial t}= & -\boldsymbol{\nabla} \cdot\left[\boldsymbol{u} n\left(\boldsymbol{x}_{i}, \hat{p}_{j}\right)\right] \\
& +\left[b(\hat{p}) 4 \pi \hat{p}^{2} f(\hat{p})\right]_{\hat{p}_{j-1}}^{\hat{p}_{j}}+Q\left(\boldsymbol{x}_{i}, \hat{p}_{j}\right),
\end{aligned}
$$

where the first term on the right-hand side describes advective transport and $Q\left(x_{i}, \hat{p}_{j}\right)$ represents the source term, $i\left(\boldsymbol{x}_{i}, \hat{p}\right)$, integrated in the $j$ th bin. Finally, $b(\hat{p}) \equiv d p / d t$ describes mechanical and radiative loss terms (see Miniati 2001 for further details on this). The most effective among these are Coulomb losses in the low-energy end and synchrotron and IC emission at the highest energy. Bremsstrahlung losses are less important, although they are included for completeness. Losses due to Coulomb collisions are (Strong \& Moskalenko 1998)

$$
\begin{aligned}
\left(\frac{d \hat{p}}{d t}\right)_{\mathrm{Coul}}= & 1.1 \times 10^{-12} \\
& \times\left\{1+\left[\ln \left(1+\hat{p}^{2}\right)^{1 / 2}-\ln n\right] \frac{1}{73.56}\right\} n \mathrm{~s}^{-1},
\end{aligned}
$$

where $n$ is the number density of the background gas in $\mathrm{cm}^{-3}$. Bremsstrahlung losses are defined as (Ginzburg 1979 Strong \& Moskalenko 1998)

$$
\left(\frac{d \hat{p}}{d t}\right)_{\text {brem }}=1.4 \times 10^{-16}\left\{\ln \left[2\left(1+\hat{p}^{2}\right)^{1 / 2}\right]-\frac{1}{3}\right\} \hat{p} n \mathrm{~s}^{-1} .
$$

For synchrotron emission and IC scattering, the combined contribution is given by

$$
\left(\frac{d \hat{p}}{d t}\right)_{\mathrm{sync}+\mathrm{IC}}=1.36 \times 10^{-20}\left(1+\frac{u_{B}}{u_{\mathrm{cmb}}}\right) \hat{p}^{2} \mathrm{~s}^{-1},
$$

where $u_{B}$ is the energy density in magnetic field, and $u_{\mathrm{cmb}}=$ $4.2 \times 10^{-13}(1+z)^{4}$ ergs $\mathrm{cm}^{-3}$ is the energy density in the cosmic microwave background.

Since our simulation treats the magnetic field passively, it is necessary to normalize the magnetic field strength. For this purpose, we assume that the volume-averaged magnetic field in a Coma-like cluster of $\sim 8 \mathrm{keV}$ is of the order of 3 $\mu \mathrm{G}$, which corresponds to values inferred from Faraday rotation measures (Kim et al. 1989, 1990). Thus, for most of the groups/clusters in our simulation, characterized by a 
temperature $T_{\mathrm{X}} \lesssim 3 \mathrm{keV}$, the energy density in the magnetic field is smaller than that in the cosmic microwave background, and the radiative loses due to IC process dominate over synchrotron losses. The IC cooling time of CR electrons is of the order of

$$
\tau_{\text {IC }}=2.3 \times 10^{8}\left(\frac{\hat{p}}{10^{4}}\right)^{-1} \mathrm{yr},
$$

where $\hat{p}=\gamma \sim 300\left(10^{4}\right)$ is required for electrons generating IC emission in the EUV (HXR) band.

\subsection{Calculation of Emissivity}

In this section we described the procedure adopted for the calculation of IC emission in the HXR and EUV bands, as well as radio synchrotron at various wavelengths of interest.

\subsubsection{Inverse-Compton Emission}

IC emission is computed in units of ergs $\mathrm{cm}^{-3} \mathrm{~s}^{-1} \mathrm{~Hz}^{-1}$ $\mathrm{sr}^{-1}$, according to (e.g., Rybicki \& Lightman 1979, but note the different units)

$$
\begin{aligned}
j_{\mathrm{IC}}(v)= & \frac{3 c \sigma_{\mathrm{T}}}{4} h v \int v_{\mathrm{cmb}}\left(v_{0}\right) d \ln v_{0} \\
& \times \sum_{j=1}^{N_{p}} f_{j} \int_{\hat{p}_{j-1}}^{\hat{p}_{j}} g\left(\frac{v}{4 \hat{p}^{2} v_{0}}\right) \hat{p}^{-q_{j}} d \hat{p},
\end{aligned}
$$

where $g(x)=2 x \ln x-2 x^{2}+x+1, v_{\text {cmb }}\left(v_{0}\right)=u_{\text {cmb }}\left(v_{0}\right) / h v_{0}$ indicates the number density of photons in the radiation field at frequency $v_{0}$, and the sum extends over all the bins of the simulated electron populations defined in equation (5). Equation (10) is the actual formula that we used to calculate the IC emission at various wavelengths from the simulated electrons. Since most of the emission is contributed from the CR electrons in the $k$ th bin such that $\hat{p}_{k-1} \leq$ $\left(v / 4 v_{0}\right)^{1 / 2} \leq \hat{p}_{k}$, for the sake of illustration we also write (Rybicki \& Lightman 1979)

$$
j_{\mathrm{IC}}(v) \simeq f_{k} \frac{8 \pi^{2} h r_{0}^{2}}{c^{2}}\left(\frac{k T_{\mathrm{cmb}}}{h}\right)^{\left(q_{k}+3\right) / 2} L\left(q_{k}-2\right) v^{-\left(q_{k}-3\right) / 2},
$$

where

$$
L(q)=2^{q+3} \frac{q^{2}+4 q+11}{(q+3)^{2}(q+5)(q+1)} \Gamma\left(\frac{q+5}{2}\right) \sum_{n=1}^{\infty} n^{-(q+5) / 2} .
$$

Thus, from equation (11) we infer that the frequency dependence of the IC emissivity is a power law, $j(v) \propto v^{-\alpha}$, with spectral index

$$
\alpha \simeq \frac{q_{k}-3}{2}
$$

\subsubsection{Synchrotron Radiation}

Analogously, for a piecewise power-law distribution of electrons given in equation (5), the synchrotron emissivity (in units of ergs $\mathrm{cm}^{-3} \mathrm{~s}^{-1} \mathrm{~Hz}^{-1} \mathrm{sr}^{-1}$ ) is computed according to the expression

$$
\begin{aligned}
j_{\mathrm{syn}}= & \frac{\sqrt{3} e^{3} B}{2 m_{e} c^{2}} \\
& \times \sum_{j=1}^{N_{p}} f_{j} p_{j}^{q_{j}}\left(\frac{2 v}{3 v_{B}}\right)^{-\left(q_{j}-3\right) / 2} \int_{x_{j-1}}^{x_{j}} F(x) x^{-\left(q_{j}-5\right) / 2} d x,
\end{aligned}
$$

where $v_{B}=e B /\left(2 \pi m_{e} c\right), x_{j}=1.5\left(v / v_{B}\right) \hat{p}_{j}^{-2}$, and we approximate $F(x)=0.87 x^{1 / 3} \exp \left(-11 x^{7 / 8} / 8\right)$ (Enßlin et al. 1999). As for the inverse Compton emission, we can also derive an approximate expression (Ginzburg \& Syrovatskii 1965),

$$
\begin{aligned}
j_{\mathrm{syn}} \simeq & f_{k} \frac{\sqrt{3} e^{2} v_{B}}{2 c\left(q_{k}-1\right)} \Gamma\left(\frac{q_{k}}{4}+\frac{13}{12}\right) \\
& \times \Gamma\left(\frac{q_{k}}{4}-\frac{7}{12}\right)\left(\frac{2 v}{3 v_{B}}\right)^{-\left(q_{k}-3\right) / 2},
\end{aligned}
$$

where $k$ refers to the momentum bin such that $\hat{p}_{k-1} \leq$ $\left(4.5 v / v_{B}\right)^{1 / 2} \leq \hat{p}_{k}$. Again, from equation (15) we infer a spectral index $\alpha \simeq\left(q_{k}-3\right) / 2$, as for the IC emission case. The local magnetic field strength, entering $v_{B}$, is given by the simulated value after a global renormalization is performed according to the criterion given in $\S 2.4$. Further, by using the morphology of the simulated field, the degree of polarization of the synchrotron radiation is also computed.

\subsection{Data Analysis}

From the simulation results, collapsed objects have been identified by the DM-based "spherical overdensity" method described in Lacey \& Cole (1994). The details of our identification procedure can be found in Miniati et al. (2000). Global quantities, $Q$ (e.g., luminosity, photon flux, spectral index), are extracted by integrating or averaging over the group/cluster volume as

$$
Q=\sum_{i} q_{i} w_{i},
$$

where $q_{i}$ is the cell value of the quantity of interest and $w_{i}$ is the weight function, i.e., the portion of computational cell within $V=4 \pi R_{\mathrm{cl}}^{3} / 3$. Values of $w_{i}$ have been computed up to a diameter of 13 computational cell, corresponding, given our current resolution, to radius $R_{\mathrm{cl}} \simeq 1.3 h^{-1} \mathrm{Mpc}$. Some of these global quantities are studied as a function of the group/cluster core temperature, $T_{\mathrm{x}}$. The latter is defined as the average temperature within a central region of diameter $0.5 h^{-1} \mathrm{Mpc}$, the typical size of the observed X-ray clusters (and over which the temperature is observationally determined). A temperature dependence is evaluated in general by fitting a computed global quantity, $Q$, to the power law

$$
Q=K\left(\frac{T_{\mathrm{X}}}{6.72 \mathrm{keV}}\right)^{\phi},
$$

where $K$ and $\phi$ are the fitting parameters. The best-fit parameters are then retrieved by a least $\chi^{2}$ analysis. Global quantities such as the luminosity at different wavelengths are expected to correlate with $T_{\mathrm{X}}$, since the emitted radiation should depend on the available group/cluster gravitational energy. In any case, we use the values of the scaling parameters to make predictions about the quantities under investigation and to make comparisons between the predictions from our simulation and observations.

Alternatively, two-dimensional maps have been constructed from the data set, either simply as slices through the volume of a physical quantity (e.g., density) or by way of a line-of-sight integration of a volume emissivity relative to a selected simulated group/cluster. The latter procedure produces synthetic surface brightness maps in the optically thin approximation through a projection code (Tregillis 2001). For comparison purposes, the groups/clusters are set to a luminosity distance corresponding to the redshift of the 
Coma Cluster $(z=0.023)$, i.e., about $70 h^{-1} \mathrm{Mpc}$. Since our resolution amounts to $\sim 200 h^{-1} \mathrm{kpc}$ per computational cell, at this distance the minimal size of a pixel of the synthetic image corresponds to $9.8 \mathrm{arcmin}^{2}$. These images allow a more in-depth inspection of the spatial distribution of the quantities of interest, but for practical reasons are limited so far to only a few samples.

\section{RESULTS}

\subsection{Inverse-Compton}

IC emission from the simulated CR electrons is computed in the following wavebands: in HXR, between 20 and 80 $\mathrm{keV}$, where measurements have been carried out for the Coma Cluster (Fusco-Femiano et al. 1999), A2256 (FuscoFemiano et al. 2000), and A3667 (Fusco-Femiano et al. 2001), and between 0.13 to $100 \mathrm{keV}$, relevant for A2199 (Kaastra et al. 1999); and in the EUV between 65 and 248 $\mathrm{eV}$, the waveband of the Extreme Ultraviolet Explorer $(E U V E)$ satellite.

Surface brightness maps for one of the collapsed objects generated in the simulation are presented in Figure 1 for the HXR emission between 20 and $80 \mathrm{keV}$ in units of $2 \times 10^{-4}$ ergs $\mathrm{cm}^{-2} \mathrm{~s}^{-1}$ per pixel and in Figure 2 for the EUV emission in units of $4 \times 10^{-7}$ ergs cm $\mathrm{cm}^{-2} \mathrm{~s}^{-1}$ per pixel. In each figure, left and right panels correspond to secondary and primary electrons, respectively. From Figures 1 and 2 it appears that the radiation from primary electrons is more extended, less centrally peaked, and morphologically more irregular than that from secondary electrons. The irregularity of the maps corresponding to the primary electrons is caused by the fact that the latter are preferentially found around existing shocks, where they are freshly injected and have not yet been depleted by radiative losses. Such irregularity is stronger for HXR than for EUV photon energies. In fact, primary HXR-emitting electrons, with Lorentz factors $\gamma \sim 10^{4}$ (cf. eq. [9]), have very short cooling times and do not propagate far from the shock where they have been generated. For the primary EUV-emitting electrons, the cooling time is about 100 times longer, and in fact these electrons are distributed throughout the collapsed object, although they are still more numerous around shocks. The distribution of secondary electrons, on the other hand, and the ensuing morphology of IC emission maps, are much less affected by, but not totally insensitive to, the presence of shocks. Their emissivity roughly varies as $n_{\text {gas }}^{2}$, indicating that although spread more widely, the distribution of the parent CR protons follows more or less that of gas (Miniati et al. 2001). Such general properties of the emission from primary and secondary electrons will characterize the radio maps as well.

The total IC emission in the wavebands of interest has been computed for each simulated group/cluster and plotted as a function of its core temperature, $T_{\mathrm{X}}$. In Figure 3 we report the fluxes of HXR radiation in the ranges 0.13$100 \mathrm{keV}$ (left panels) and 20-80 keV (right panels) produced by secondary (top) and primary (bottom) electrons, respectively, for a luminosity distance of the simulated groups/ clusters of $70 h^{-1}$ Mpc. In Figure 4 we present the luminosity in the EUV band (65-248 eV) for secondary (left) and primary (right) electrons. The values reported for the primary electrons assume $R_{e / p}=1$ (see $\S 2.2$ for further details). The data plots have been fitted to a power-law correlation as described in $\S 2.6$. The best-fit parameters are given in Table 1. Note that with primary electrons the slope $\phi$ for $20-80 \mathrm{keV}$ HXR is somewhat smaller than those for 0.13-100 keV HXR and EUV. This is at least partly because of larger scatter seen in the bottom right panel of Figure 3. We believe that this scatter has a physical origin in the different shock-acceleration history undergone by each collapsed object identified in the simulation box. However, we
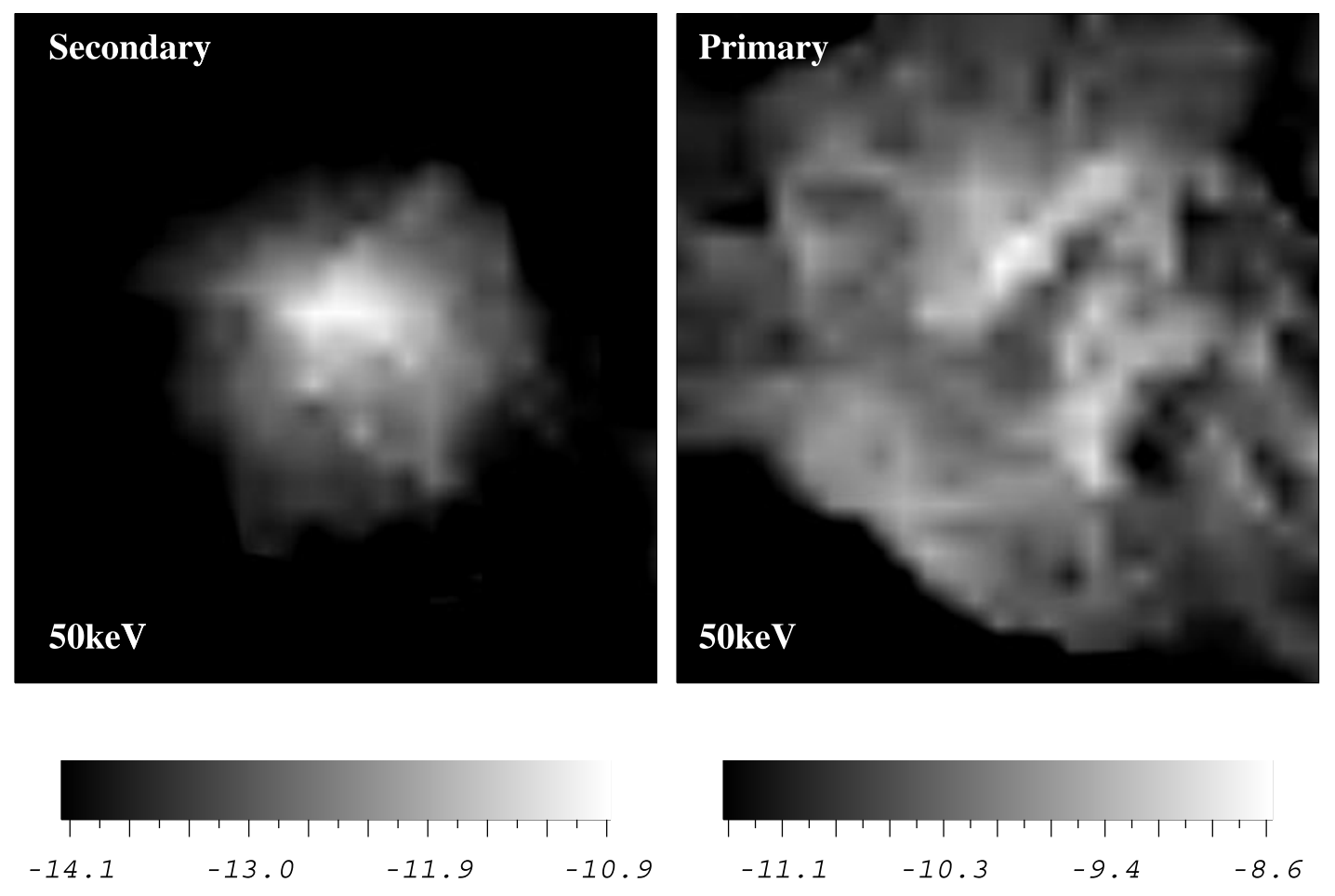

FIG. 1.-Emission map of HXR in units of $2 \times 10^{-4} \mathrm{ergs} \mathrm{cm}^{-2} \mathrm{~s}^{-1}$ per pixel, from secondary $(l e f t)$ and primary (right) electrons at $50 \mathrm{keV}$ for one of the collapsed objects generated in the simulation. The linear size of the panels corresponds to $5.5 h^{-1} \mathrm{Mpc}$. 

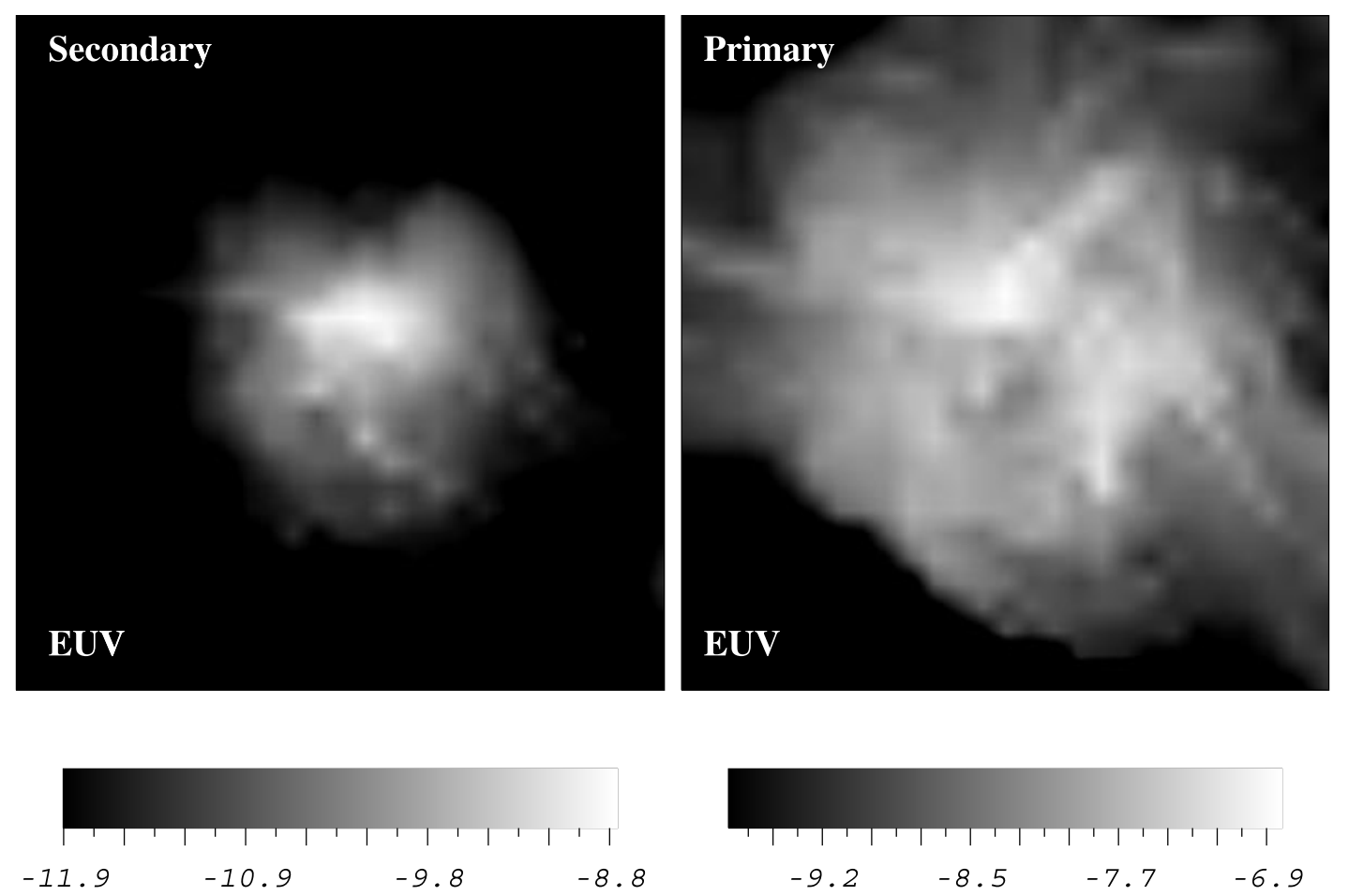

FIG. 2.-Emission map in units $4 \times 10^{-7}$ ergs $\mathrm{cm}^{-2} \mathrm{~s}^{-1}$ per pixel, of EUV radiation for the same object as in Fig. 1. Left and right panels correspond to secondary and primary electrons, respectively. The linear size of the panels correspond to $5.5 h^{-1} \mathrm{Mpc}$.

do expect larger simulations, which allow both more numerous and larger clusters as well as higher resolution, to result in more consistent values of $\phi$.

The temperature scaling of the emitted radiation from the two CR electron populations is quite different. This fact corresponds to differences in the scaling properties of secondary and primary electrons with the group/cluster temperature. The scaling of the IC flux from secondaries is expected to go as

$$
F_{\text {IC }}(v) \propto N_{\text {es }}\left(\gamma_{v}\right) \propto N_{\text {CR }}\left(\gamma_{v}\right) n_{\text {gas }},
$$

where $N_{\text {es }}\left(\gamma_{v}\right)$ and $N_{\mathrm{CR}}\left(\gamma_{v}\right)$ are the total number of secondary electrons with energy $\gamma_{v}$ responsible for the emission at the specified frequency, $v$, and that of the parent CR ions, respectively. By accounting for the gas accretion rate and for the injection mechanism adopted here as roughly converting a fixed fraction of the shocked gas into CRs (see $\S 2.2$ ), it can be shown that $N_{\mathrm{CR}}\left(\gamma_{v}\right) \propto T_{\mathrm{X}}^{2}$. In addition, the average baryon density inside the group/cluster volume within the fixed radius of $1.3 h^{-1} \mathrm{Mpc}$ has an almost linear dependence on $T_{\mathrm{X}}$, in agreement with observations (Edge \& Steward 1991; Mohr, Mathiesen, \& Evrard 1999). Although resolution effects discussed in $\S 2.1$ may cause some steepening of the $F_{\mathrm{IC}}-T_{\mathrm{X}}$ relation, our numerical results are consistent with the above arguments. On the other hand, for the IC emission from primary electrons we have

$$
F_{\text {IC }}(v) \propto N_{\text {ep }}\left(\gamma_{v}\right) \sim N_{\mathrm{CR}}\left(\gamma_{v}\right),
$$

since the total number of primary electrons and ions should be proportional at the time of injection. Therefore, in this case the temperature dependence of the flux from IC scattering should be weaker by roughly one power of $T_{\mathrm{X}}$ with respect to the secondary CR electrons model, as indeed we have found.

We have also studied the spectral properties of the radiation emitted by the simulated electrons. Since the published data report observed values of the spectral index for the HXR emission only, we only present our results for this waveband, although the same general discussion described below applies to EUV-emitting electrons as well.

TABLE 1

\begin{tabular}{|c|c|c|c|c|}
\hline \multirow[b]{2}{*}{ ENERGY BAND } & \multicolumn{2}{|c|}{ SECONDARY $e^{-}$} & \multicolumn{2}{|c|}{ PRIMARY $^{\mathrm{a}} e^{-}$} \\
\hline & $K^{\mathrm{b}}$ & $\phi$ & $K^{\mathrm{b}}$ & $\phi$ \\
\hline$F_{\text {IC }}$ HXR: $20-80 \mathrm{keV} \ldots \ldots \ldots$ & $2.8 \times 10^{-13}$ & 2.9 & $8.0 \times 10^{-11}$ & 1.5 \\
\hline$F_{\text {IC }}$ HXR: $0.13-100 \mathrm{keV} \ldots \ldots$ & $7.0 \times 10^{-12}$ & 2.9 & $3.6 \times 10^{-9}$ & 1.9 \\
\hline$L_{\mathrm{IC}}$ EUV: $65-248 \mathrm{eV} \ldots \ldots \ldots$ & $1.5 \times 10^{41}$ & 2.9 & $1 \times 10^{44}$ & 1.9 \\
\hline$P_{1.4 \mathrm{GHz}} \ldots \ldots \ldots \ldots \ldots \ldots \ldots \ldots \ldots \ldots$ & $5.1 \times 10^{23}$ & 4.2 & $2.1 \times 10^{26}$ & 2.6 \\
\hline$P_{330 \mathrm{MHz}} \ldots \ldots \ldots \ldots \ldots \ldots \ldots \ldots$ & $1.6 \times 10^{24}$ & 4.1 & $6.1 \times 10^{26}$ & 2.6 \\
\hline$P_{74 \mathrm{MHz}} \cdots \cdots \cdots \cdots \cdots \cdots \cdots \cdots$ & $8 \times 10^{24}$ & 4.2 & $2 \times 10^{27}$ & 2.7 \\
\hline$P_{31 \mathrm{MHz}} \ldots \ldots \ldots \ldots \ldots \ldots \ldots \ldots$ & $2 \times 10^{25}$ & 4.2 & $4.3 \times 10^{27}$ & 2.8 \\
\hline
\end{tabular}

Temperature SCALing Relations

a The values reported in the table for primary electrons assume $R_{e / p}=1$.

${ }^{\mathrm{b}}$ Units are: For $F_{\mathrm{IC}}$ : $\operatorname{ergs~s}^{-1} \mathrm{~cm}^{-2}$; for $L_{\mathrm{IC}}: \operatorname{ergs~s}{ }^{-1}$; for $P: \mathrm{W} \mathrm{Hz}^{-1}$. 
IC-HXR Flux
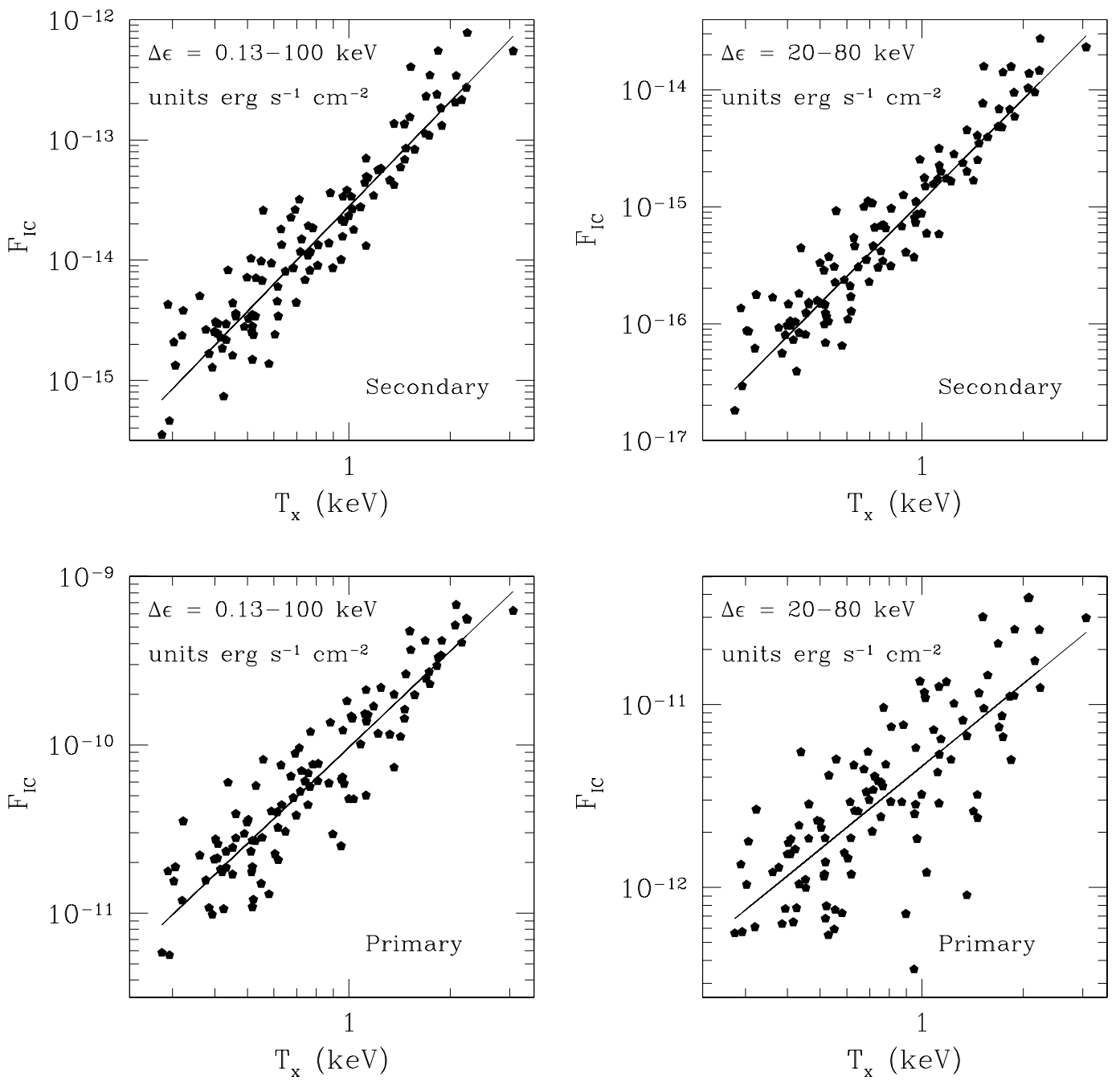

FIG. 3.-Group/cluster HXR flux of IC emission in $0.13-100 \mathrm{keV}($ left $)$ and in $20-80 \mathrm{keV}$ (right) due to secondary (top) and primary electrons (bottom), as a function of the group/cluster core temperature. The emissivity is integrated over a spherical volume within a radius of $1.3 h^{-1}$ Mpc. Note that the physical values of the luminosities for primary electrons should be scaled with the electron to proton injection ratio, $R_{e / p}$.

EUV Luminosity
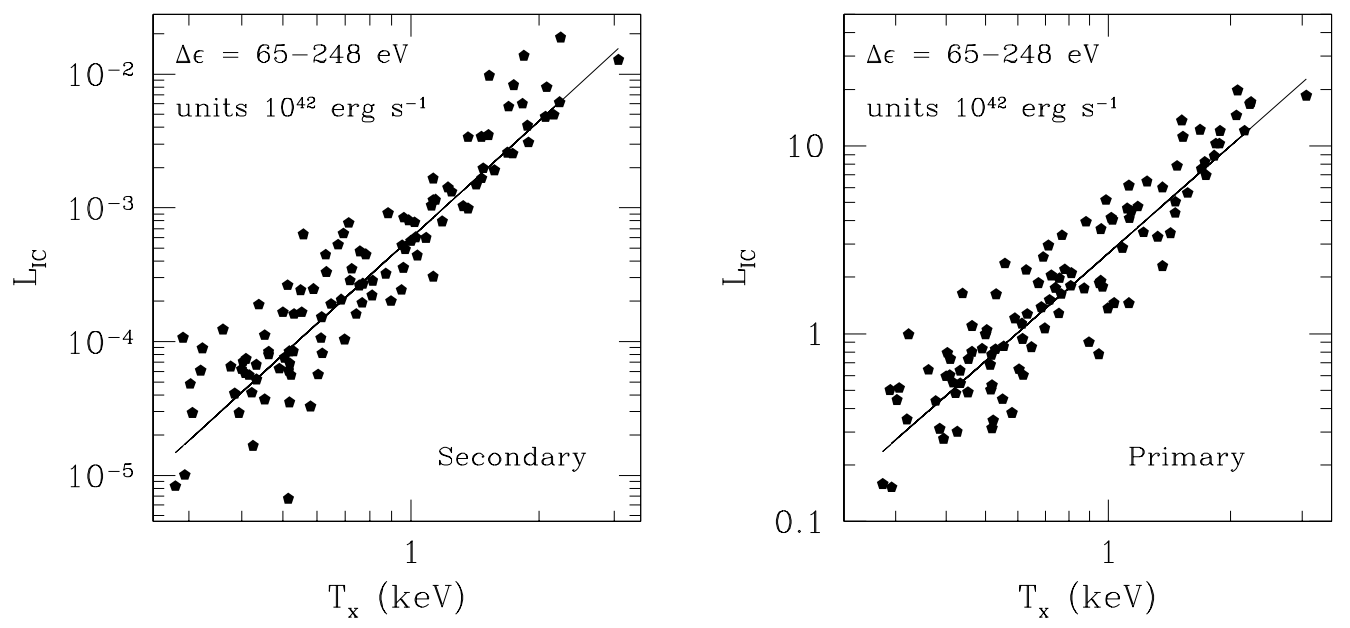

FIG. 4.-Group/cluster EUV luminosity from IC emission due to secondary electrons (left) and primary electrons (right) as a function of the group/cluster core temperature. The emissivity is integrated over a spherical volume within a radius of $1.3 \mathrm{~h}^{-1} \mathrm{Mpc}$. Note that the physical values of the luminosities for primary electrons should be scaled with the electron to proton injection ratio, $R_{e / p}$. 
Thus, for each simulated group/cluster we have computed the IC HXR spectral index, $\alpha_{\mathrm{IC}}$, averaged over a clustercentered volume defined by a radius $R$ to give $\bar{\alpha}_{\mathrm{IC}}(<R)$. Our results are reported in Figure 5 for both primary (bottom panels) and secondary electrons (top panels), as a function of the clusters/groups temperature, $T_{\mathrm{X}}$, for four different values of $R$.

As for the primary electrons, the situation is complicated because our spatial resolution is inadequate to follow in detail the spectral evolution of this population of CR electrons immediately downstream of shocks. In fact, after being shock-accelerated, CR electrons will rapidly radiate away their energy as they are advected away from shockacceleration regions. Thus, in a realistic picture, at various distances from the shock we should find populations of CR electrons with increasingly "older age." However, for initial energies of interest the timescale for cooling is typically shorter than the time for advection across a numerical cell. Therefore, the proper CR electrons spatial distribution cannot be reproduced because of resolution limits. Nevertheless, we know that for the case of IC and synchrotron cooling, the summation of all of these "aging" populations in the postshock region produces a volume-averaged population with a power-law index steeper by 1 than that produced at the shock. Thus, the spectral index of the volume-averaged emission from the postshock region can be recovered from the properties of the shock. In addition, in order to compute correctly the total emission from the postshock region, we adopt a subcycling procedure that allows us to estimate the steady-state number density of high-energy electrons there.

With this clarification, in the four bottom panels of Figure 5 we present the volume-averaged spectral index associated with the postshock IC emission region. According to our discussion above, the spectral index characterizing the IC emission from the high-energy electrons that are emerging from the acceleration region, i.e., those immediately behind the shock, is flatter by about 0.5 (cf. eq. [13]). For shocks with Mach number $\geq 3$, representing most of the shocks in our simulation, the spectral index is $\alpha_{\mathrm{IC}} \leq 1.3$. Indeed, in the last panel (bottom right) of Figure 5, corresponding to an averaging volume of radius $1.3 \mathrm{~h}^{-1} \mathrm{Mpc}$
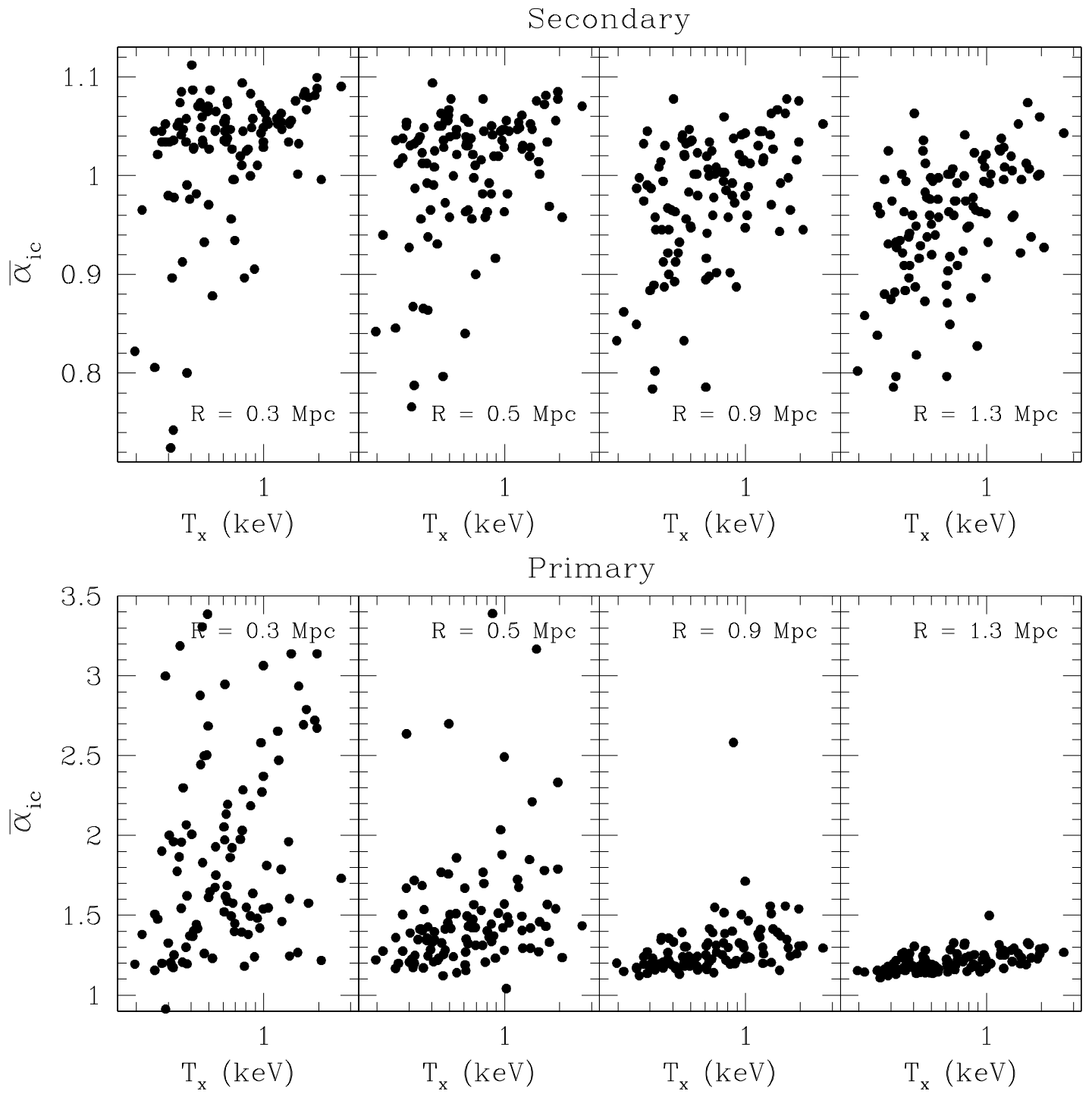

FIG. 5.-Top: Volume-averaged spectral index for the HXR radiation at $50 \mathrm{keV}$ from secondary electrons as a function of the group/cluster core temperature for four different averaging volume radii, i.e., from left to right, $R=0.3,0.5,0.9,1.3 h^{-1} \mathrm{Mpc}$. Bottom: Same as top, but now for primary electrons. 
around the group/cluster center, we have $\bar{\alpha}_{I C} \simeq 1.0-1.3$. This volume is large enough to include most of the shocks where fresh particles are injected. On the other hand, for smaller radii, most of the considered volume has been depleted of energetic particles through radiative losses. Therefore, we find higher values and larger fluctuations for $\bar{\alpha}_{\mathrm{IC}}(<R)$.

Now turning our attention to the top panels of Figure 5, we note that for the secondary electrons $\bar{\alpha}_{\text {IC }}(<R)$ mostly ranges between 0.8 and 1.1. The distribution of secondary electrons, $f_{\text {es }}$, is determined by the loss equation, which reads

$$
\frac{\partial}{\partial t} f_{\mathrm{es}}(\hat{p})=\frac{1}{\hat{p}^{2}} \frac{\partial}{\partial \hat{p}}\left[\hat{p}^{2} f_{\mathrm{es}}(\hat{p}) b(\hat{p})\right]+i(\hat{p}),
$$

where $\hat{p}$ and $b(\hat{p})$ have been previously defined and $i(\hat{p})$ is the term describing the source of particles. For a steady-state solution, if $i(\hat{p}) \propto \hat{p}^{-r}$ and $b(\hat{p}) \propto \hat{p}^{t}$, then the particle distribution is

$$
f_{\mathrm{es}}(\hat{p}) \propto \hat{p}^{-r+1-t} .
$$

We point out that most of the CR ions produced in our simulation have a spectrum characterized by $q_{i}=4.0-4.2$ (Miniati et al. 2001), which translates into a slope for the source function of secondary electrons close to the same value, i.e., $r \simeq 4.2$. For IC losses, relevant for the HXR emitting electrons, $t=2$; thus, from equation (13),

$$
\alpha_{\mathrm{IC}}=\frac{(r-1+t)-3}{2} \simeq 1.1,
$$

in rough accord with the values in the top plots of Figure 5. We note, however, in those plots the presence of several points indicating values of $\bar{\alpha}_{\mathrm{IC}}(<R)<1$. In addition, the number of such points becomes more numerous as we move toward the right panel, i.e., larger averaging volumes (Fig. 5). These values of the spectral index would imply the presence of secondary electrons with distributions, $f_{\text {es }}(p) \propto p^{-q_{\text {es }} \text {, }}$ flatter than $q_{\mathrm{es}}=r-1+t=4-1+2=5$, i.e., than our simulated CR ions (with $q_{i} \simeq 4$ ) can generate. Such distributions are in fact produced by the reacceleration that some secondary electrons undergo as they encounter a shock wave in the ICM. This event is more likely to happen at the outskirts of a cluster, where shocks are more frequent, in agreement with the trend of the top panels in Figure 5. However, since we expect such reaccelerated secondary CR electrons to behave exactly as the primary electrons discussed above, the flattening of the spectral index should be only "visible" very close to the shocks and should disappear when the radiation from the large postshock emission region is considered. Thus, as a result of the same coarse grid effects described above in the context of primary electrons, the spectral indexes reported in the top panels of Figure 5 tend to be smaller than they should. Unlike the case of the primary electrons, however, correcting for this effect is highly impractical because it would require following (numerically) the reaccelerated particles as an independent population. In any case, we expect the values of $\bar{\alpha}_{\text {IC }}$ $(<R)$ reported in the panel corresponding to an averaging volume of $R=0.3 \mathrm{Mpc}$ to be only little affected by the reaccelerated populations of secondary CR electrons, and therefore fairly representative of the simulation results.

Finally, we point out that for EUV-emitting secondary electrons, with Lorentz factor $\gamma \sim 300$, the loss mechanism are not only IC emission but also Coulomb collisions, implying a smaller value for both $t$ and $\alpha$.

\subsubsection{Hard $X$-Ray Emission}

Having computed the fitting parameters summarized in Table 1, we can now estimate the HXR fluxes for the Coma, A2199, and A2256 clusters as predicted by our simulation model. For this exercise, an extrapolation has been applied using the parameters in Table 1, since the collapsed objects identified in simulation data have temperatures $\lesssim 3 \mathrm{keV}$, while the observed clusters used for comparison have higher temperatures. In order to define the scope of such extrapolations, first we note that the plots reported in Table 1 show quite a bit of (real) scatter, which we attribute to the different evolution of the collapsed objects. Thus, our plots will only allow an estimate of average values and within a factor of the order of the scatter. As pointed out above, the scatter in the bottom right panel of Figure 3 is particularly large. With this clarification, in Table 2 we report the name of each observed cluster in the first column, and the measured redshift, temperature, HXR flux and spectral index in columns (2)-(5), respectively (references in col. [10]). The

TABLE 2

Flux of HXR Radiation: Measurements versus Predictions

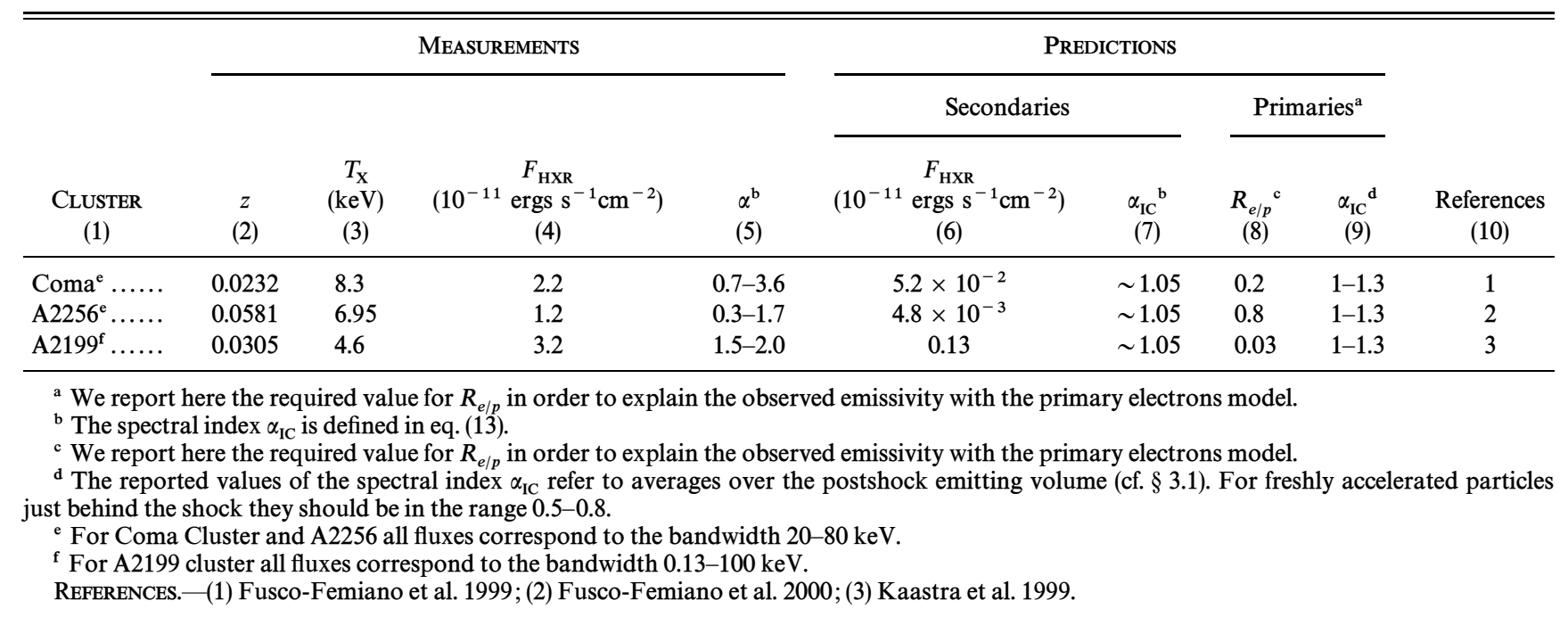


expected average HXR flux and spectral index according to our secondary model are reported in columns (6) and (7), respectively. Finally, columns (8) and (9) refer to the primary CR electrons model; the latter lists the spectral index associated with each population of CR particles and the former gives the $R_{e / p}$ value required in order for the HXR flux from primary CR electrons to match the reported observations.

The values reported in Table 2 indicate that the total number of secondary electrons is too small to account for the full measured HXR flux from either Coma, A2256, or A2199. In fact, the calculated emission from secondary electrons in our simulation corresponds at most to a few percent of the reported measurements. Higher values of the total injection efficiency $\eta_{\text {inj }}$ (see $\S 2.2$ ) could allow a larger population of $\mathrm{CR}$ ions and, therefore, of secondary electrons. However, the resulting nonthermal pressure and $\gamma$-ray flux associated with the CR ions would then become inconsistent with the observational limits (Miniati et al. 2001; Blasi \& Colafrancesco 1999; Blasi 1999).

On the other hand, in order to account for reported detections in the HXR waveband with the emission from the primary electrons in our simulation would require $R_{e / p} \gtrsim 0.2$ for Coma and Abell 2256 and $R_{e / p} \gtrsim 0.03$ for Abell 2199. Since measurements for galactic CRs give $\boldsymbol{R}_{e / p} \sim 0.01-0.05$ (Mueller \& Tang 1987), it would appear that the only case of Abell 2199 could be explained in terms of IC emission from primary electrons. However, as already pointed out, our plot in the bottom right panel of Figure 3 exhibits a large scatter, of about 1 order of magnitude around the average values at each temperature, because of the different accretion/merger status of the simulated group/cluster. Thus the HXR emission from the Coma Cluster and Abell 2256 is also consistent with the primary model, if a major accretion/merger process occurred in the recent history of these clusters, as indeed indicated by the observations (Briel et al. 1991; Burns et al. 1994).

Regarding the spatial distribution of the HXR radiation, it is unknown for the Coma Cluster (Fusco-Femiano et al. 1999), but there is some indication that it may be more widely distributed than the thermal emission for A2199 (Kaastra et al. 1999), as would result from the IC process
(Sarazin \& Lieu 1998). The emission from simulated secondary CR electrons is smoother and less extended than that from primary CR electrons, but the available information on the spatial distribution of detected HXR emission is not sufficient yet to discriminate observationally between the two alternative models.

\subsubsection{Extreme-Ultraviolet Emission}

Regarding the EUV radiation, in Table 3 we compare the published EUV excess measurements in column (3) with our numerical results for the EUV luminosity due to IC emission from secondary CR electrons in column (4), and the value of $R_{e / p}$ required to match the observations for primary CR electrons in column (5).

The values computed in Table 3 for a cluster with the same temperature as Coma indicate that both primary and perhaps even secondary CR electrons could produce through IC emission an EUV radiation flux comparable to the measurement reported by Bowyer et al. (1999). Our numerical model, however, falls short of the detected emission from the Virgo Cluster (Berghoefer, Bowyer, \& Korpela 2000; Lieu et al. 1996b) by about 2 orders of magnitude, although the presence of M81 may induce higher nonthermal particle populations in the ICM of Virgo and enhance the emission in this waveband. For the remaining two clusters, A1795 and A2199, our numerical model for both primary (assuming $R_{e / p} \sim 10^{-2}$ ) and secondary electrons indicates a luminosity of the order of $10^{41}-10^{42}$ ergs $\mathrm{s}^{-1}$, well below the reported detections. The 2 order of magnitude difference is significant even considering the scatter in the reported plots and the limitations in our work. So, for these two clusters we suspect that the reported EUV excesses are unlikely to be produced by IC scattering off cosmic microwave background photons of CR electrons shock-accelerated in the ICM. This conclusion is further supported by the following insight. The simulation results in Figure 4 show a clear trend of the EUV luminosity increasing with the temperature of the collapsed object for both primary and secondary electrons. Such a trend is not present in the observational data. According to Table 3, the reported EUV luminosity for both A1795 and A2199 is larger by about 2 orders of magnitude than the value

TABLE 3

EMISSION OF EUV Radiation IN THE EUV BAND 65-248 eV: MEASUREMENTS VERSUS PREDICTIONS

\begin{tabular}{|c|c|c|c|c|c|}
\hline \multirow[b]{2}{*}{$\begin{array}{c}\text { CLUSTER } \\
\text { (1) }\end{array}$} & \multirow[b]{2}{*}{$\begin{array}{c}T_{\mathrm{X}} \\
(\mathrm{keV}) \\
(2)\end{array}$} & \multirow[b]{2}{*}{$\begin{array}{c}\text { MEASUREMENTS } \\
\left(\mathrm{ergs} \mathrm{s}^{-1}\right) \\
\text { (3) }\end{array}$} & \multicolumn{2}{|c|}{ Predictions $^{\mathrm{a}}$} & \multirow[b]{2}{*}{$\begin{array}{c}\text { REFERENCES } \\
\text { (6) }\end{array}$} \\
\hline & & & $\begin{array}{c}\text { Secondaries } \\
\left(\operatorname{ergs~s}^{-1}\right) \\
\text { (4) }\end{array}$ & $\begin{array}{l}R_{e / p} \\
(5)\end{array}$ & \\
\hline \multirow[t]{2}{*}{ Coma (A1656)...... } & 8.3 & $1.5 \times 10^{42}$ & $2.7 \times 10^{41}$ & $1 \times 10^{-2}$ & 1 \\
\hline & 8.3 & $5.0 \times 10^{43}$ & $2.7 \times 10^{41}$ & 0.3 & 2 \\
\hline \multirow{2}{*}{ Virgo ............... } & 1.8 & $5.2 \times 10^{42}$ & $3.2 \times 10^{39}$ & 0.6 & 3 \\
\hline & 1.8 & $9.0 \times 10^{42}$ & $3.2 \times 10^{39}$ & 1 & 2 \\
\hline \multirow[t]{2}{*}{ A1795 ............. } & 5.8 & $\ldots$ & $1 \times 10^{41}$ & $\ldots$ & 1 \\
\hline & 5.8 & $2.0 \times 10^{45}$ & $1 \times 10^{41}$ & 27 & 4 \\
\hline \multirow[t]{2}{*}{ A2199 .............. } & 4.5 & $\ldots$ & $4.5 \times 10^{40}$ & $\ldots$ & 1 \\
\hline & 4.5 & $5.0 \times 10^{43}$ & $4.5 \times 10^{40}$ & 1 & 5 \\
\hline
\end{tabular}

${ }^{a}$ We report here the required value for $R_{e / p}$ in order to explain the observed emissivity with the primary electrons model.

REFERENCES.- (1) Bowyer et al. 1999; (2) Lieu et al. 1996b; (3) Berghoefer et al. 2000; (4) Mittaz et al. 1998; (5) Sarazin \& Kempner 2000. 
reported for Coma by Bowyer et al. (1999). This contradicts the theoretical trends shown in our plots and cannot be accounted for by the scatter among the simulated groups/ clusters. We note that this peculiar feature of the observational results will affect any model in which the EUV radiation is produced by energetic electrons generated through general cluster evolution. In fact, whether accelerated by accretion shock waves, merger shock waves, intracluster turbulence, or generated in hadronic interactions, the energy content of the relativistic electrons can always be expressed as a fraction of the total energy of the cluster (which scales with its temperature).

The synthetic EUV images can also be compared to the thermal X-ray and synchrotron radio emission maps displayed on the same scale in Figures 9 and 10, below. As we can see, the EUV emissivity from both primary and secondary electrons is more extended than the thermal X-rays, as observed in real clusters. However, the EUV radiation in these IC-based models is also more extended than the radio emissivity; this contradicts observational results, as first pointed out by Bowyer \& Berghoefer (1998). Thus, the morphological properties of the emitting region pose another challenge to our attempt to attribute the EUV excess to CR electrons of cosmological origin.

\subsection{Synchrotron Radiation}

The synchrotron radiation emitted by $\mathrm{CR}$ electrons has been calculated using equation (14). We remind the reader that we have assumed for this calculation a volumeaveraged magnetic field in a Coma-like cluster of the order of $3 \mu \mathrm{G}$ and an emitting volume of radius $1.3 h^{-1} \mathrm{Mpc}$. Figure 6 displays the simulated radio spectral power, $P_{v}$, at $1.4 \mathrm{GHz}$ (left) and $330 \mathrm{MHz}$ (right) from secondary (top) and primary (bottom) electrons, as a function of the group/ cluster temperature. For each plot, the best-fit parameters for a power-law correlation (cf. $\$ 2.6$ ) are reported in Table 1. Although the emitting electrons are approximately the same as those producing HXRs, the temperature dependence of $P_{v}$ is steeper now, reflecting the additional scaling of the magnetic field with the group/cluster temperature.

\section{Synchrotron Power}
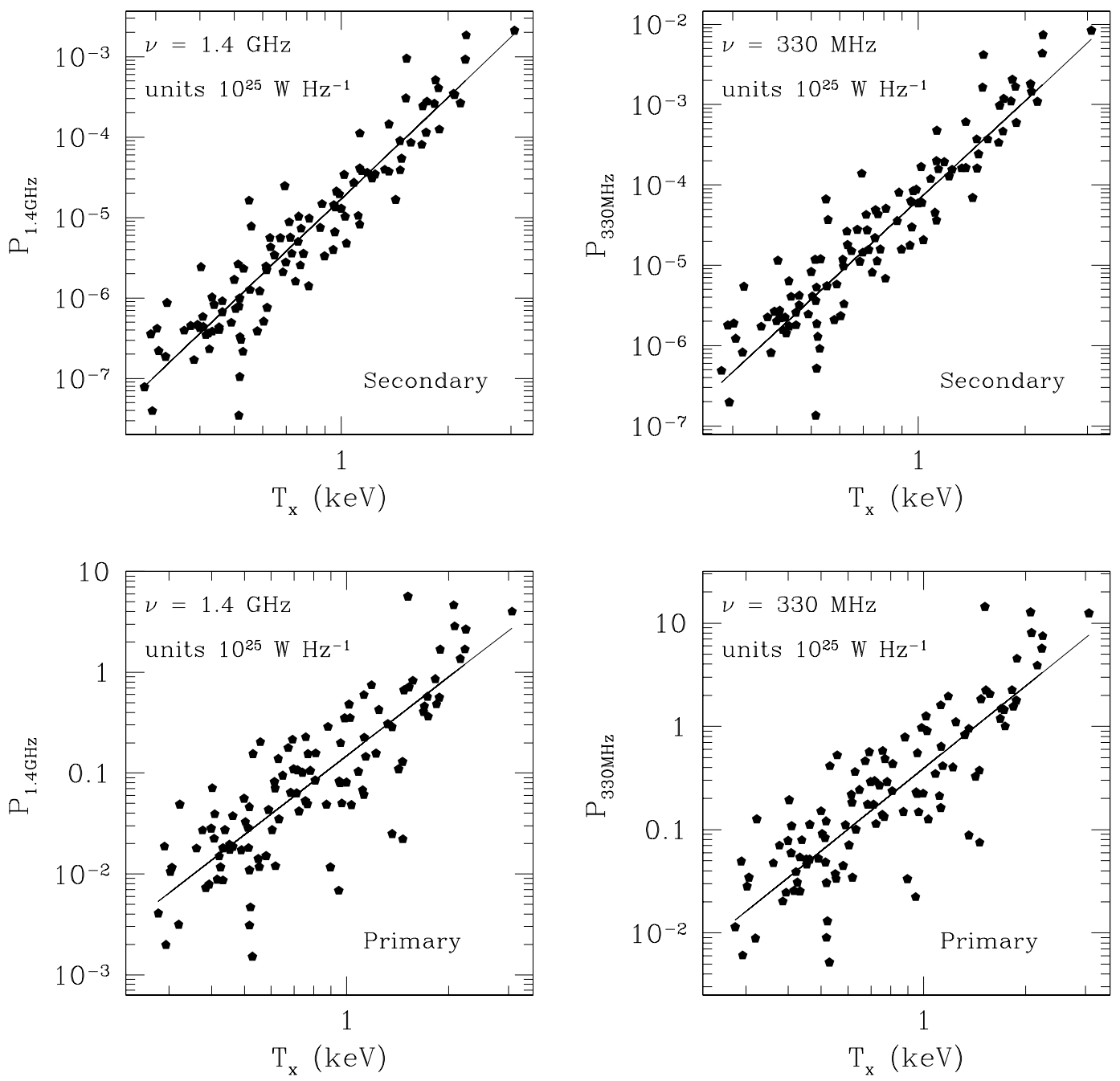

FIG. 6.-Group/cluster synchrotron power at $1.4 \mathrm{GHz}$ (top left) and at $330 \mathrm{MHz}$ (top right) from secondary electrons and at $1.4 \mathrm{GHz}$ (bottom left) and at $330 \mathrm{MHz}$ (bottom right) from primary electrons as a function of the core temperature. The emissivity is integrated over a spherical volume within a radius of $1.3 h^{-1} \mathrm{Mpc}$. Note that the physical values of the luminosities for primary electrons should be scaled with the electron to proton injection ratio, $R_{e / p}$. 
The latter is close to $T_{\mathrm{X}}^{0.75}$ (see $\S$ 3.2.1) in our simulation, and indeed the values of $\phi$ have increased by approximately 1 unit with respect to the IC scattering emission.

By analogy with the HXR emission, we have calculated the volume-averaged synchrotron spectral indices for radiation at $1.4 \mathrm{GHz}$ and $31 \mathrm{MHz}$, which bracket the range of most commonly observed frequencies. These spectral indices are illustrated in Figure 7 for secondary electrons and in Figure 8 for the primary electrons. As for the HXR case, the spectral index associated with the emission from primary electrons represents the value averaged over the postshock emitting volume. As discussed at length in $\S 3.1$, the spectral index of the CR electrons just behind the acceleration region should be smaller by one-half with respect to what reported in those figures. First, those plots do not show any relevant difference between values of the spectral index at the frequencies of $1.4 \mathrm{GHz}$ and $31 \mathrm{MHz}$, in the case of either primary or secondary electrons. This indicates that within the energy range that correspond to the above frequencies, there is no break due to radiative losses in the particle distribution. Similarly to the case of the HXR IC emission and after making analogous considerations, we find $\bar{\alpha}_{\text {syn }}(<R) \gtrsim 1.1$ for the secondary electrons and $1-1.3$ for the primary electrons in the large averaging volume that contains the acceleration sites. Again, this is because the particles responsible for the emission in the two cases (radio and HXR) represent approximately the same populations.

\subsubsection{Secondary Electrons and Radio Halos}

The secondary electrons from our numerical simulation can account for a number of features observed in radio halos, including the morphology of the emitting region, the polarization properties, and the temperature dependence of the synchrotron power at $1.4 \mathrm{GHz}$ (Liang et al. 2000).

In Figure 9 we show synthetic maps of the surface brightness from synchrotron radiation from secondary electrons for the same object as in Figure 1. In each of the three panels we present the radio emission at different wavelengths, namely, $1.4 \mathrm{GHz}$ (top left), $330 \mathrm{MHz}$ (top right), and
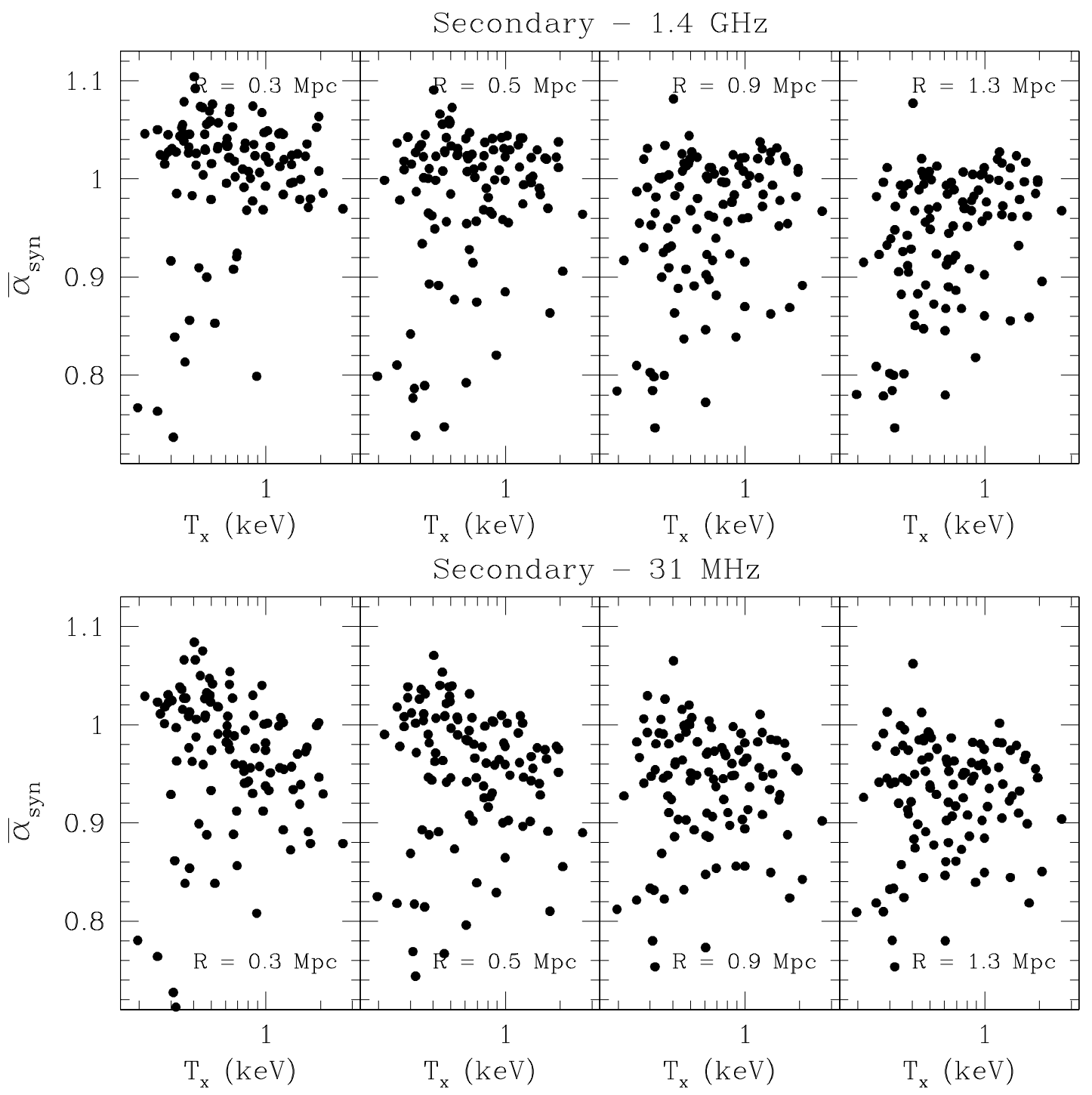

FIG. 7.-Volume-averaged spectral index for the synchrotron radiation from secondary electrons at $1.4 \mathrm{GHz}$ (top) and $31 \mathrm{MHz}$ (bottom) as a function of the group/cluster core temperature. The four panels in each row correspond to the four different averaging volume radii (left to right) $R=0.3,0.5,0.9,1.3 h^{-1}$ Mpc. 

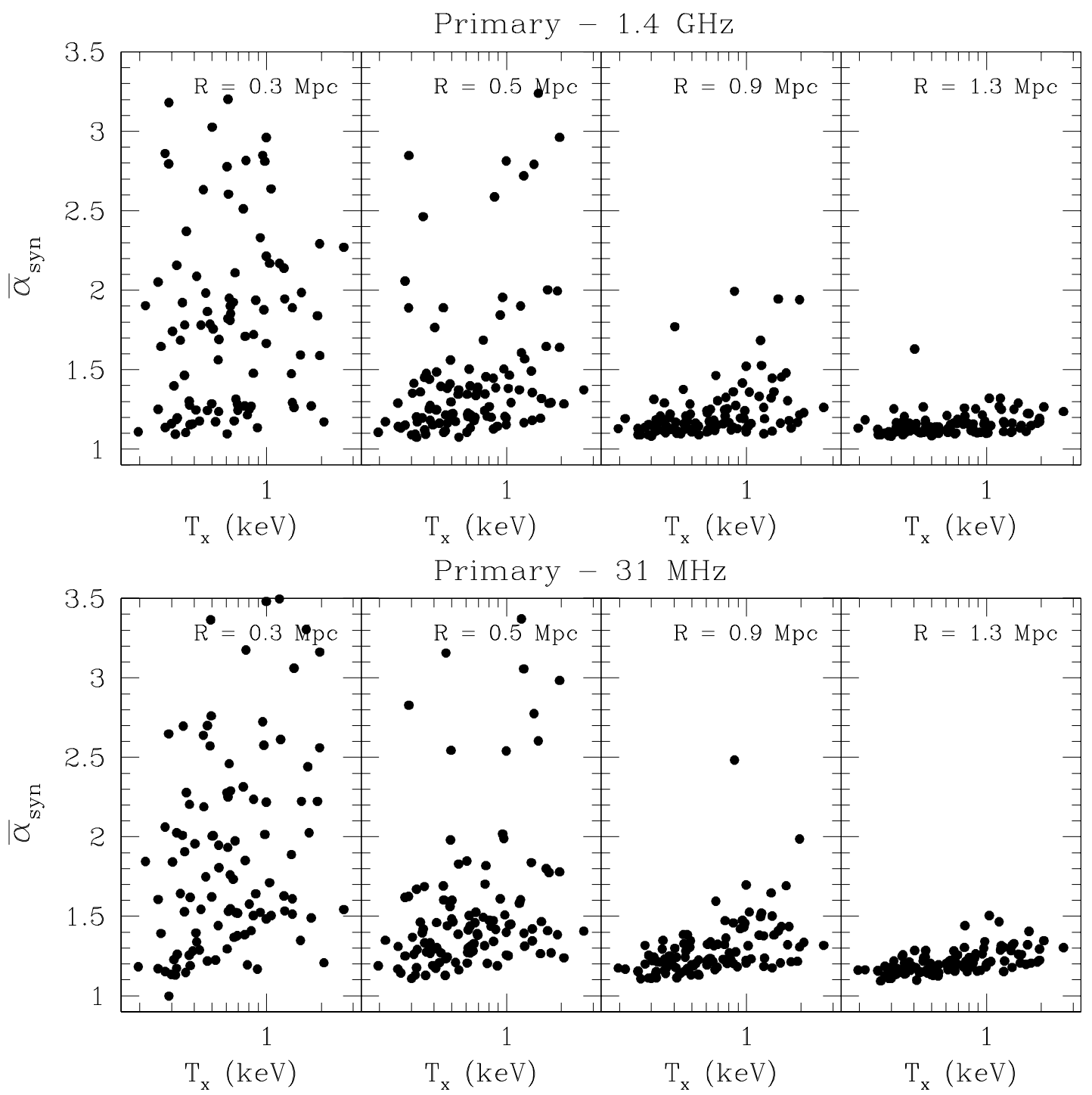

FIG. 8.-As in Fig. 7, but for primary electrons

$31 \mathrm{MHz}$ (bottom left). For reference, we also show the X-ray map produced by the thermal bremsstrahlung. The radio emission extends throughout the X-ray group/cluster, indeed resembling the spatial features of observed radio halos. By quantitative analysis of the emission map, the lower frequency emission appears slightly more extended, again in agreement with the observations. This trend is due to the decay of the magnetic field strength at large distances from the cluster centers and the fact that, according to equation (15), the emissivity dims first at higher frequencies. A comparison with the images of X-ray from thermal bremsstrahlung reveals a higher level of substructure in the radio emission than in the thermal emission. Such substructure resembles to some extent that seen in the synthetic $\gamma$-ray images of Miniati et al. (2001) and is connected to the underlying shock distribution in the ICM. However, the synchrotron map will also depend on the structure of the magnetic field, which in fully resolved simulations or in real clusters might extend to regions smaller than we have been able to simulate here. In any case, radio spatial features are also seen in real observations. There, the region responsible for the radio emission often appears irregular, and in this sense shows some departure from the observed X-ray image (e.g., A665, Giovannini \& Feretti 2000; A520, Govoni et al. 2001; and $\mathrm{Cl} 0016+16$, Giovannini \& Feretti 2000).

According to our calculations, a typical Coma-like cluster $\left(T_{\mathrm{X}}=8.3 \mathrm{keV}\right)$ should exhibit a radio power at 1.4 $\mathrm{GHz}$ of the order of $1.5 \times 10^{24} \mathrm{~W} \mathrm{~Hz}^{-1}$, close to the observations (see Table 4). We caution that this result depends on our choice of the magnetic field normalization of a few $\mu \mathrm{G}$ for a Coma-like cluster. The significance of this finding is that the energy supplied by structure shocks would suffice to power radio halos if magnetic fields of a few $\mu \mathrm{G}$ exist inside such clusters. In addition, our numerical findings indicate a scaling of $P_{1.4 \mathrm{GHz}} \propto T^{4.2}$, in good agreement with the observational values (Liang et al. 2000). In fact, the synchrotron power in the secondary model should scale as

$$
\begin{aligned}
P_{\mathrm{syn}} & \propto N_{\mathrm{es}} B^{1+\alpha_{\mathrm{syn}}} \propto N_{\mathrm{CR}} n_{\mathrm{gas}} \frac{B^{1+\alpha_{\mathrm{syn}}}}{B^{2} / 8 \pi+u_{\mathrm{cmb}}} \\
& \propto T^{3} T^{2 \phi_{B}} \propto T^{3+2 \phi_{B}},
\end{aligned}
$$



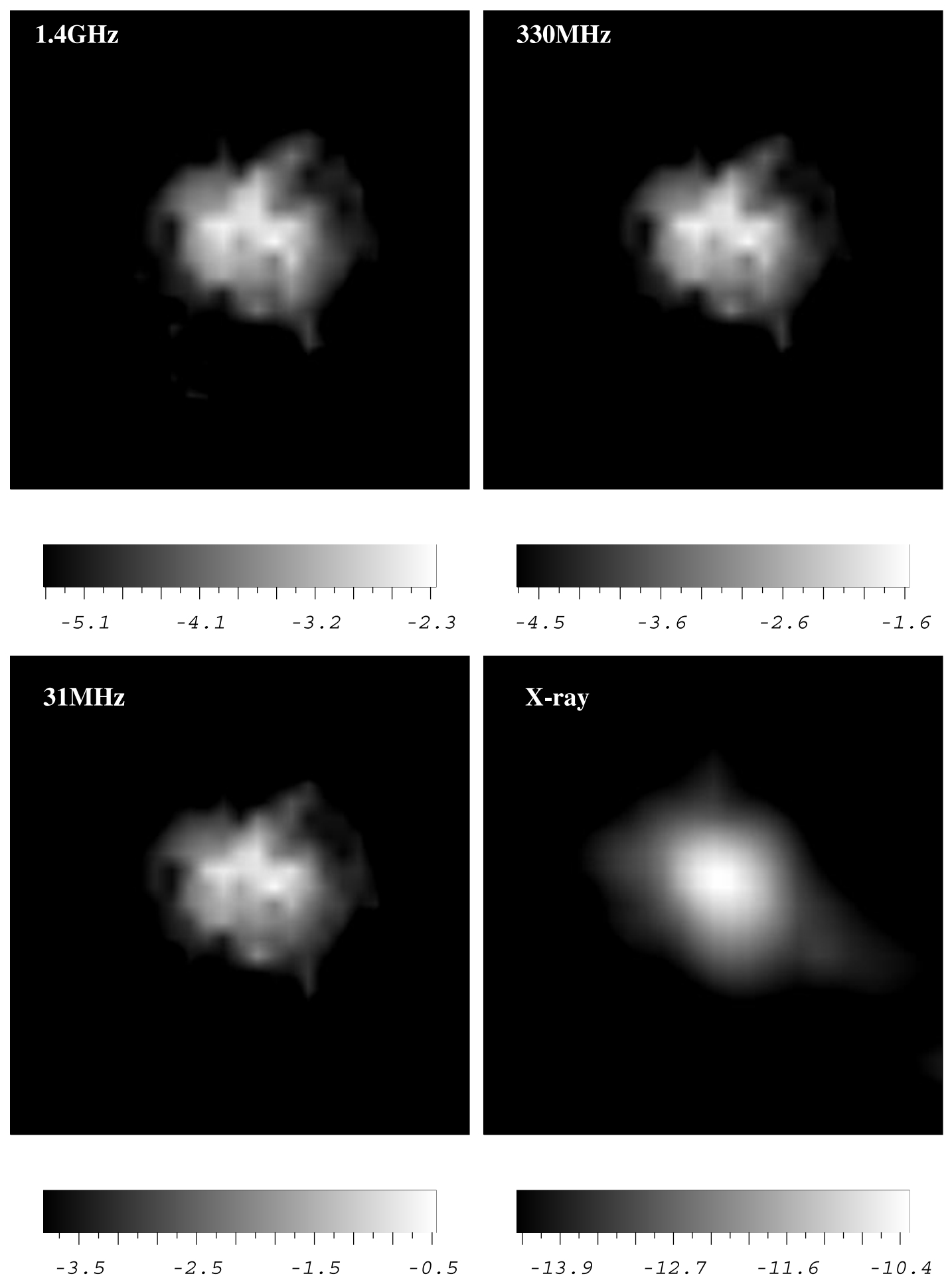

FIG. 9.- Radio map of surface brightness in units of Jy per pixel $\left(1 \mathrm{Jy}=10^{-23} \mathrm{erg} \mathrm{cm}^{-2}\right)$ for the same object as in Fig. 1 , at $1.4 \mathrm{GHz}($ top left $), 330 \mathrm{MHz}$ (top right), and $31 \mathrm{MHz}$ (bottom left). The radiation is synchrotron emission generated by secondary electrons in the group/cluster magnetic field. For comparison we also show the thermal X-ray map (bottom right) in units of ergs $\mathrm{cm}^{-2} \mathrm{~s}^{-1}$. The linear size of the panels correspond to $5.5 h^{-1} \mathrm{Mpc}$.

where $\alpha_{\text {syn }} \simeq 1$ is the radio spectral index, and $N_{\mathrm{es}} \propto$ $N_{\mathrm{CR}} n_{\mathrm{gas}} /\left(B^{2} / 8 \pi+u_{\mathrm{cmb}}\right)$ is the number of secondary electrons, proportional to the product of the of CR ions and the gas density. The factor $1 /\left(B^{2} / 8 \pi+u_{\mathrm{cmb}}\right)$ simply accounts in a steady state for the reduction of secondary electrons from radiative losses. In addition, $\phi_{B}$ indicates parametrically the temperature dependence of the magnetic field strength, i.e.,
$B \propto T^{\phi_{B}}$. In $\S 3.1 .1$ we already saw that $N_{\text {es }} \propto N_{\text {CR }} n_{\text {gas }} \propto$ $T^{3}$. In addition, from our simulation we have found $\phi_{B} \sim$ 0.75 . The amplification of the magnetic field is primarily due to shear flows developed during large-scale structure formation and, to a lesser extent, to gas compression. The accuracy of the magnetic field strength dependence on the group/cluster temperature in the simulation is not clear, 
TABLE 4

Radio Power at $1.4 \mathrm{GHz}$ : Measurements versus Predictions of SECONDARY ELECTRONS MODEL

\begin{tabular}{|c|c|c|c|}
\hline Quantity & Measurements & Model & References \\
\hline$P_{1.4 \mathrm{GHz}}(8.3 \mathrm{keV})^{\mathrm{a}} \ldots \ldots$ & 1.5 & 1.2 & 1 \\
\hline 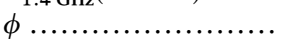 & $4-5.2$ & $\sim 4.2$ & 2 \\
\hline$\alpha_{\text {syn }} \quad b \ldots \ldots \ldots \ldots \ldots \ldots$ & $0.5-\leq 1.6$ & $\gtrsim 1.1$ & 1,3 \\
\hline Polarization $(\%) . . . . .$. & $\leq 10$ & A few & 4 \\
\hline
\end{tabular}

a The radio power is measured in units of $10^{24} \mathrm{~W} \mathrm{~Hz}^{-1}$. Here we compare the normalizations of the $P_{1.4 \mathrm{GHz}}$ vs. $T_{\mathrm{X}}$ relation by taking the measured and predicted values of $P_{1.4 \mathrm{GHz}}$ for the Coma cluster.

${ }^{b}$ The range includes values for different clusters as well as spatial variations inside the same cluster.

REFERENCES. - (1) Deiss et al. 1997; (2) Liang et al. 2000; (3) Hanish 1982; (4) Feretti 1999.

since the $B-T_{\mathrm{X}}$ relation is observationally unknown, although now under investigation (P. P. Kronberg 2001, private communication). Clearly, however, the coarse grid effects limit the amount of shear and reduce the growth of the field, as has already been pointed out in analogous studies (Roettiger, Stone, \& Burns 1999b; Dolag, Bartelmann, \& Lesch 1999).

With the above temperature scalings of $N_{\mathrm{es}}$ and $B$, the result $P_{1.4 \mathrm{GHz}} \propto T^{4.2}$ is roughly retrieved. However, since $\alpha_{\text {syn }} \sim 1$, when $B \geq\left(8 \pi u_{\text {cmb }}\right)^{1 / 2}(1+z)^{2} \simeq 3.3 \mu \mathrm{G}$, the synchrotron power becomes independent of the magnetic field strength, and its dependence on the cluster temperature inevitably flattens. For the temperature-scaling properties of the magnetic field in our simulation and the adopted normalization, such a turnover should occur at a temperature above $9.4 \mathrm{keV}$ (at $z=0$ ). Given the poor statistics of real clusters with temperatures beyond this value, conclusions in this respect are premature.

We have also investigated the fractional polarization that characterizes the integrated synchrotron emission in the synthetic maps. From our investigation we find that the degree of polarization is very low throughout the simulated groups/clusters, typically of the order of a few percent. This result holds for the radiation at $1.4 \mathrm{GHz}$ and $330 \mathrm{MHz}$. In this calculation, no Faraday depolarization was included. Its inclusion would further reduce the degree of polarization found here (Dolag \& Enßlin 2000), reinforcing our conclusions below. The low degree of polarization of synchrotron emission is one of the distinctive features of observed radio halos. The outcome in our model is probably due to the highly complex structure of the magnetic field inside the group/cluster. In fact, the radiation from different depths along the line of sight will imprint a polarization angle determined by the integrated structure of the field. Since the field is a varying function of space, the sum of all the contributions along one line of sight and orthogonal field projections of equal magnitude lead to a null net polarization, and the emergent radiation from a disordered field will be mostly unpolarized.

Another important quantity that is determined by the observations is the spectral index of the radiation. In the secondary CR electron model this should be $\gtrsim 1.1$. For the case of the Coma Cluster, the best-studied example, the integrated radio flux below $1.4 \mathrm{GHz}$ is well fitted by a spectral index 1.16-1.3 (Deiss et al. 1997). This is marginally consistent with our results, and in fact our current model underestimates the radio emission of the Coma Cluster at $330 \mathrm{MHz}$ with respect to the data of Venturi, Giovannini, \&
Feretti (1990). In addition, the observed spectral index shows a steepening with increasing radius. No conclusion can be drawn from our plots in this respect. In fact, although Figure 7 shows that the emission-averaged spectral index tends to decrease slightly when larger volumes are considered, this is a spurious effect, as was discussed in $\S 3.1$. In general, the observational values of the spectral index may be slightly higher than what we predict (Hanish 1982). In the context of secondary electrons from cosmic shocks, this would correspond to slightly weaker shocks in the ICM than those typically found within group/cluster ICMs in our simulation. In fact, there is a possible tendency for the Mach numbers we compute to be slightly overestimated, especially in regions close to the cluster core, where with moderate spatial resolution the shock compression can be "confused" by the gravitational compression (see Miniati et al. 2000 for details). Although a precise estimate should be carried out through higher resolution simulations, a simple estimate indicates that

$$
\Delta \alpha=\frac{\Delta q}{2}=-1.5 \frac{\gamma_{\mathrm{gas}}+1}{\left(1-M^{-2}\right)^{2}} \frac{1}{M^{3}} \Delta M,
$$

where $\gamma_{\text {gas }}=5 / 3$ is the adiabatic index of the gas. Therefore, an error of $\Delta M \sim 1$ in the Mach number estimate translates into an error of $\Delta \alpha \sim-0.2$ for the spectral index, which would allow for spectra steeper than reported in our plots.

\subsubsection{Primary Electrons}

In Figure 10 we present synthetic maps of the surface brightness due to synchrotron emission from primary electrons at $1.4 \mathrm{GHz}$ (top left), $330 \mathrm{MHz}$ (top right), and $31 \mathrm{MHz}$ (bottom left) for the same object as in Figure 9. In the bottom right panel, we also present on the same scale a two-dimensional cut of the contour levels of shock compression in a plane through the center of the collapsed object and perpendicular to the line of sight of the other three panels. In contrast to the emission produced by the secondary electrons, the primary electron emission maps are strikingly inhomogeneous, with the primary electrons (and emission) strongly concentrated around shocks. Figure 10 resembles the HXR images presented in Figure 1 (right), which also refers to the same object.

The location of these synchrotron emission regions at the immediate outskirts of the group/cluster, together with their irregular and extended morphology, may suggest a connection with the observed radio relics. The suggestion is reinforced by the finding that the fractional polarization through the extended regions is quite high, of the order of $30 \%-50 \%$, comparable to the values suggested by the observations. Unlike the secondary electrons, the primary electrons produce emission primarily in the immediate postshock regions. There, the magnetic field is highly aligned as a consequence of the enhancement of the perpendicular component by the shock compression. Thus, the radiation suffers only minor structural depolarization along the line of sight and emerges highly polarized. We do point out that our results might overestimate the polarization expected in real clusters because finite resolution limits the possible complexity of the magnetic field structure.

In Table 5 we present a comparison between the observed properties of radio relics for a few sources and the averaged values predicted in our numerical work. To define the scope of this comparison, we point out again that the predictions of our model, illustrated in the bottom panels of 

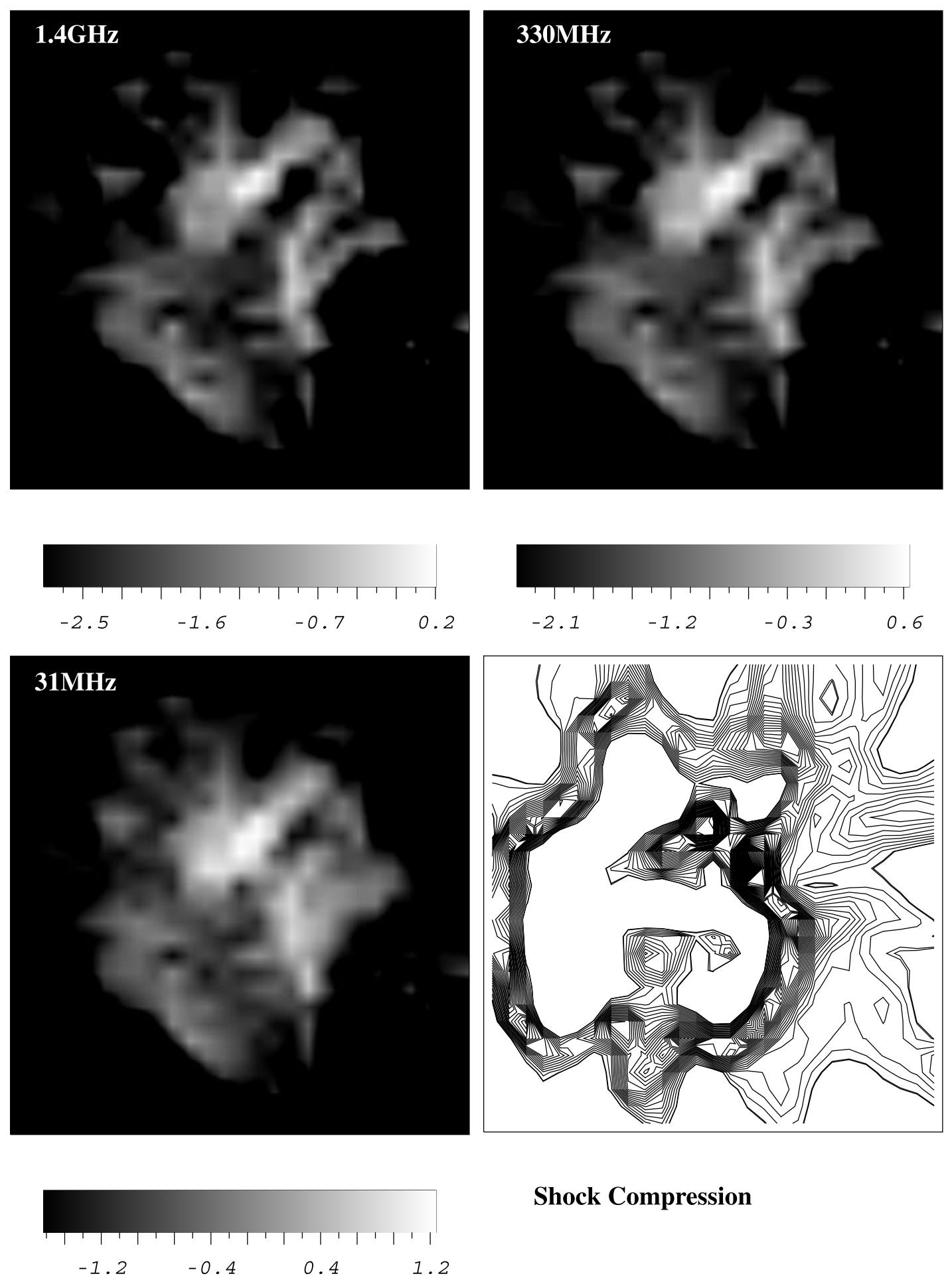

Shock Compression

FIG. 10.- Same as Fig. 9, but for primary electrons in the top left, top right, and bottom left panels. A two-dimensional cut of the contour levels of shock compression in a plane through the center of the collapsed object is also shown in the bottom right panel, for comparison.

Figure 6, indicate a range of values that span a factor of a few about some average. With this in mind, we note that in all the presented cases the mean predicted total power at 1.4 $\mathrm{GHz}$ is consistent with the observed values for a conservative estimate of $R_{e / p}$ of $10^{-2}$ to $10^{-3}$. This result signifies that, again, the power supplied at shocks by accretion flows is sufficient to generate the emission observed in extended radio relics, for conservative values of shock acceleration injection and efficiency.
We have also calculated the volume-averaged spectral index (cf. § 3.1) for each pixel in the images of Figure 10. We find that in the brightest regions $\bar{\alpha}_{\text {syn }} \sim 1-1.3$ at both 1.4 $\mathrm{GHz}$ and $31 \mathrm{MHz}$. Where the emissivity is low, the spectral index steepens to values $\bar{\alpha}_{\text {syn }} \approx 1.4-1.5$ (at $31 \mathrm{GHz}$ ). These values are typical for all the groups/clusters analyzed with the simulation data (Miniati 2000) and are indeed consistent with the observed values for relics, a few of which are reported in Table 5. In addition, the above volume-averaged 
TABLE 5

Properties of Radio Relics: Measurements versus Predictions of Primary Electrons Model

\begin{tabular}{|c|c|c|c|c|c|c|c|}
\hline \multirow[b]{2}{*}{ ReLIC } & \multirow[b]{2}{*}{ Cluster } & \multirow[b]{2}{*}{ FREQUENCY } & \multicolumn{3}{|c|}{ MEASUREMENT } & \multirow[b]{2}{*}{$\begin{array}{c}\text { MODEL }^{\mathrm{a}} \\
R_{e / p}^{\mathrm{b}}\end{array}$} & \multirow[b]{2}{*}{ REFERENCES } \\
\hline & & & $\begin{array}{c}\text { Flux } \\
\left(\mathrm{Jy}=10^{-23} \mathrm{ergs} \mathrm{cm}^{-2}\right)\end{array}$ & $\begin{array}{l}\text { Polarization } \\
\quad(\%)\end{array}$ & $\alpha$ & & \\
\hline \multirow[t]{2}{*}{$1253-275 \ldots \ldots \ldots \ldots$} & Coma & $1.4 \mathrm{GHz}$ & 0.16 & $25-30$ & $1.1 \pm 0.2^{\mathrm{c}}$ & $1 \times 10^{-3}$ & 1,2 \\
\hline & & $330 \mathrm{MHz}$ & 1.4 & $\ldots$ & $1.1 \pm 0.2$ & $4 \times 10^{-3}$ & 2 \\
\hline $2006-56 \ldots \ldots \ldots \ldots$ & A3667 & $1.4 \mathrm{GHz}$ & 0.7 & $\ldots$ & $0.5-1.2$ & $6 \times 10^{-2}$ & 3,4 \\
\hline Component G...... & A2256 & $1.4 \mathrm{GHz}$ & 0.15 & 20 & $0.8 \pm 0.1$ & $1 \times 10^{-2}$ & 5 \\
\hline Component H...... & A2256 & $1.4 \mathrm{GHz}$ & 0.1 & 20 & $0.8 \pm 0.1$ & $7 \times 10^{-3}$ & 5 \\
\hline $1140+203 \ldots \ldots \ldots$ & A1367 & $1.4 \mathrm{GHz}$ & 0.18 & $\ldots$ & $1.9 \pm 0.5$ & $7 \times 10^{-3}$ & 6 \\
\hline
\end{tabular}

${ }^{a}$ The spectral index in the model is always in the range $0.5-0.8$ for the emitting region immediately behind the shock and $1-1.3$ when the volume-average value is considered (see $\S 3.1$ for further details). Likewise, the prediction for the fractional polarization is always $30 \%-50 \%$, although geometrical effects may reduce the observed value with respect to it (Enßlin et al. 1998).

${ }^{\mathrm{b}}$ Here we report the required value for $R_{e / p}$ in order to explain the observed emissivity with the primary electrons model.

${ }^{c}$ Andernach et al. 1984 find 1.18.

REFERENCES.- (1) Venturi et al. 1990; (2) Giovannini et al. 1991; (3) Goss et al. 1982; (4) Rottgering et al. 1997; (5) Rottgering et al. 1994; (6) Gavazzi \& Trinchieri 1983.

values of $\bar{\alpha}_{\mathrm{syn}}$ corresponds to a spectral index of the order of $\sim 0.5-0.8$ for the bright emission regions associated with the freshly accelerated populations just behind the shock (cf. $\S 3.1)$. Observation of the relic source $2006-56$ is consistent with the above depiction as the spectral index increases from $\bar{\alpha}_{\text {syn }} \approx 0.5$ from the edge of the source to about $\bar{\alpha}_{\text {syn }} \approx$ 1.1 toward its center (Rottgering et al. 1997). This trend may indicate that the emitting particles in that source are indeed accelerated by shocks, as was pointed out by Enßlin et al. (1998).

\section{DISCUSSION AND CONCLUSIONS}

In this paper we have explored the acceleration, losses, and spatial transport of cosmic-ray electrons for the first time in a fully cosmological simulation of large-scale structure formation. In our simulation, CR electrons were either directly injected at shocks (primary) or generated in hadronic collisions of CR ions and the nuclei of the ICM (secondary). In general, the populations of CRs thus generated are described by power laws, $f(p) \propto p^{-q}$, with index $q$ depending on the strength of the shock. Our results indicate that the total number of relativistic electrons present in each group/cluster scales with the temperature of the ICM in the sense that more numerous populations are present in hotter groups/clusters.

Nonthermal emissions due to relativistic electrons have been computed at different wavelengths in order to address the issues related to the recent EUV/HXR observations of clusters of galaxies. As for the excess of emission in the EUV waveband, we find that the electrons generated in our simulation are probably insufficient to produce the claimed detections (Lieu et al. 1996b, 1996a; Mittaz et al. 1998; Berghoefer et al. 2000), except for the case of the Coma Cluster reported by Bowyer et al. (1999). Furthermore, the EUV excess reported so far has shown no trend with cluster temperature, contrary to the results of our simulation. The absence of such trends would suggest that the processes responsible for the generation of nonthermal electrons do not depend on the energetics of groups/clusters.

The contribution to the HXR emissivity from the secondary electrons amounts to only a few percent of the currently reported values (namely, for the Coma Cluster and A2556 in the bandwidth $20-80 \mathrm{keV}$, and for A2199 in the bandwidth $0.13-100 \mathrm{keV})$. Increasing the total number of sec- ondary electrons enough to account for all the HXR excess emission would imply a corresponding increase of parent CR ions and a consequent $\gamma$-ray flux much above the upper limits established by EGRET (Blasi \& Colafrancesco 1999; Blasi 1999; (Miniati et al. 2001). The average HXR emission from primary electrons extrapolated from our plot in the bottom right panel of Figure 3 indicates a contribution from this component of about $10 \%$ or so of the reported detections in this waveband. This plot, however, is characterized by a large spread of about an order of magnitude around the average, a reflection of the different level of shock activity in the ICM of individual groups/clusters. Thus, the reported HXR emission from the Coma Cluster and Abell 2556 could be accounted for by IC emission of primary electrons, given that these clusters are known to be, respectively, in a postmerger phase (Burns et al. 1994) or in the process of merging (Briel et al. 1991).

From the analysis of the synchrotron emission, we find that our secondary CR electrons could explain several features of radio halos; namely, the total power at $1.4 \mathrm{GHz}$, the fractional polarization, the morphology, and, marginally, even the spectral index. The radio power, $P_{1.4 \mathrm{GHz}}$, as reported by Liang et al. (2000), for clusters at different temperatures is produced by the secondary electron model, provided that the magnetic field varies with the group/cluster temperature as steeply as found in our simulation. Consistency here is achieved by assuming a normalization for the magnetic field that gives the volume-averaged value of $3 \mu \mathrm{G}$ for a Coma-like cluster, as inferred from Faraday rotation measure estimates (Kim et al. 1989, 1990). Numerous properties of radio halos are reproduced quite naturally according to our simulation, although the spectral index reported by observations is sometimes steeper than found here (see also Chi et al. 1996). Future higher resolution simulations should allow us to determine whether or not this is a numerical artifact, as suggested in $\S$ 3.2.1. A similar result for the secondary model for radio halos was recently obtained by Dolag \& Enßlin (2000), although in their case the CR population was not simulated but assumed from the beginning. In our simulation the spectra of accelerated particles are determined consistently with the cosmic shocks responsible directly or indirectly for their acceleration. Thus, particle distribution and spectra are not free parameters of the computation. 
Finally, we have shown that the primary electrons directly accelerated at cosmic shocks generate radio maps that are similar in radiated power, morphology, and polarization fraction to observed radio relics. The accelerating shocks have been shown to be strong enough to produce quite flat populations of CR electrons in the region immediately adjacent to the acceleration sites. Our limited numerical resolution prevents us from reproducing correctly the spatial distribution of electron populations of different "ages" propagating away from the shock. However, the computed flat spectra of the shock-accelerated electron distributions indicate that the volume-averaged spectral index of the whole postshock emission should be in the observed range, $\alpha=1-1.5$.

Thus the energetics of accretion/merger shocks are in principle sufficient to power both the radio-relic emission and the reported detections of HXR excess. However, in order for the same electron population to account for the emission in both wavebands for either the Coma Cluster or Abell 2256 (see Tables 2 and 4), we require a volumeaveraged magnetic field strength about an order of magnitude smaller than what is assumed here. It is conceivable that the magnetic field strength drops by this amount as we move from the cluster center to its outskirts where the accretion shocks powering both the radio relics and the HXR emission would be located according to our simulations. And in fact this is supported by the magnetic field maps of our simulations (Miniati 2000). However, because of the limited numerical resolution, such properties of the magnetic field cannot be considered as conclusive.

It is important to mention the existence of alternative models for the existence of radio relics. In particular, it has been pointed out that radio relics could be sites where fossil radio plasma, expelled by radio galaxies at some point during cosmic history, is currently being revived through the encounter with a shock wave by means of adiabatic compression (Enßlin 1999; Enßlin \& Gopal-Krisna 2001). The idea is compelling, particularly because the ejection of relativistic plasma by radio galaxies in the intergalactic medium is an observational fact. It has been shown that radio plasma up to $2 \mathrm{Gyr}$ of age can be revived by strong shocks (Enßlin \& Gopal-Krisna 2001). The statistics, i.e., the probability of actually seeing revived radio plasma at a given epoch, is not an easy task because it depends on the luminosity function of radio galaxies as well as the subsequent evolution of the radio plasma in the cosmic environment. However, future low-frequency observation should substantially improve our knowledge of the distribution of old radio plasma in the cosmic environment (Enßlin \& Gopal-Krisna 2001). Nevertheless, there exist some important differences between radio relics produced by primary electrons accelerated at shocks and those resulting from revived radio plasma. In fact, according to recent magetohydrodynamical simulations of the interaction of radio plasma with a shock wave, a cloud of radio plasma becomes unstable upon being shocked, and develops into a complex filamentary structure (Enßlin \& Brüggen 2001). It is not clear that such morphological properties belong to giant radio relics observed at the outskirts of cluster of galaxies. However, recent radio observations have shown that a sample of radio relics with sizes up to a few hundred kpc observed at very high resolution in a number of galaxy clusters possess just these morphological features (Slee et al. 2001). It is possible then that both mechanisms, namely, shock acceleration and compression of old radio plasma, are at work in the ICM, producing a large variety of radio sources with distinct properties, as outlined above. This possibility, just speculated here, should be investigated in the future.

From a broader perspective, it is very important to understand the actual origins of radio halos and relics, so that one can assess the level of nonthermal activity in the ICM. If, as provided in the present model, radio halos are produced by secondary electrons, they reveal the existence of a related population of CR ions that have developed during the formation of the large-scale structure (Völk et al. 1996; Berezinsky et al. 1997; Miniati 2000; Miniati et al. 2001). Such populations may contain a significant fraction of the total energy density of the ICM and could therefore be dynamically important (Miniati 2000; Miniati et al. 2001), with many cosmological implications.

F. M. wishes to acknowledge support from a Doctoral Dissertation Fellowship at the University of Minnesota and from a fellowship provided by the Research Training Network of the European Commission for the Physics of the Intergalactic Medium. The work of F. M. and T. W. J. has been supported by NASA grant NAG5-5055, by NSF grants AST 96-16964 and AST 00-71167, and by the University of Minnesota Supercomputing Institute. D. R. and H. K. were supported in part by grant 1999-2-113-001-5 from the interdisciplinary research program of the KOSEF. We are grateful to I. L. Tregillis for his ray-tracer code and to I. V. Moskalenko and A. W. Strong for providing their GALPROP routines. F. M. thanks Sebastian Heinz for useful comments on the manuscript and acknowledges several insightful discussion with Torsten Enßlin. Finally, we thank the referee, P. Blasi, for several constructive comments to the manuscript.
Andernach, H., Feretti, L., \& Giovannini, G. 1984, A\&A, 133, 252 Bagchi, J., Pislar, V., \& Lima Neto, G. B. 1998, MNRAS, 296, L23 Berezinsky, V. S., Blasi, P., \& Ptuskin, V. S. 1997, ApJ, 487, 529

Berghoefer, T. W., Bowyer, S., \& Korpela, E. 2000, ApJ, 535, 615 Blasi, P. 1999, ApJ, 525, 603 2000, ApJ, 532, L9

2001, Astropart. Phys. 15, 223

Blasi, P., \& Colafrancesco, S. 1999, Astropart. Phys., 12, 169

Bonamente, M., Lieu, R., \& Mittaz, J. P. 2001, ApJ, 546, 805

Bowyer, S., \& Berghoefer, T. W. 1998, ApJ, 506, 502

Bowyer, S., Berghoefer, T. W., \& Korpela, E. 1999, ApJ, 526, 592

Briel, U. G., et al. 1991, A\&A, 246, L10

Burns, J. O., Roettiger, K., Ledlow, M., \& Klypin, A. 1994, ApJ, 427, L87

Cen, R., \& Ostriker, J. P. 1999, ApJ, 517, 31

Chi, X., Young, E. C. M., Yu, P. K. N., Zang, L., \& Zhu, Q.-Q. 1996, Phys. Rev. Lett., 77, 1436

\section{REFERENCES}

Clarke, T. E., Kronberg, P. P., \& Böhringer, H. 2001, ApJ, 547, L111 Deiss, B. M., Reich, W., Lesch, H., \& Wielebinski, R. 1997, A\&A, 321, 55 Dennison, B. 1980, ApJ, 239, L93

Dermer, C. D. 1986, A\&A, 157, 223

Dixon, W. V., Sallmen, S., Hurwitz, M., \& Lieu, R. 2001, ApJ, 550, L25 Dogiel, V. A. 2000, A\&A, 357, 66

Dolag, K., Bartelmann, M., \& Lesch, H. 1999, A\&A, 348, 351

Dolag, K. \& Enßlin, T. 2000, A\&A, 362, 151

Drury, L. O. 1983, Rep. Prog. Phys., 46, 973

Edge, A. C., \& Steward, G. C. 1991, MNRAS, 252, 414

Eilek, J. A., \& Wetherall, J. C. 1999, in Diffuse Thermal and Relativistic Plasma in Galaxy Clusters, ed. H. Böhringer, L. Feretti, \& P. Schuecker (Garching: MPI), 249

Ellison, D. C., Berezhko, E. G., \& Baring, M. G. 2000, ApJ, 540, 292

Enßlin, T. A. 1999, in Diffuse Thermal and Relativistic Plasma in Galaxy Clusters, ed. H. Böhringer, L. Feretti, \& P. Schuecker (Garching: MPI), 275 
Enßlin, T. A., Biermann, P. L., Klein, U., \& Kohle, S. 1998, A\&A, 332, 395

Enßlin, T. A., Biermann, P. L., Kronberg, P. P., \& Wu, X.-P. 1997, ApJ, 477,560

Enßlin, T. A., \& Brüggen, M. 2001, MNRAS, in press

Enßlin, T. A., \& Gopal-Krisna. 2001, A\&A, 366, 26

Enßlin, T. A., Lieu, R., \& Biermann, P. L. 1999, A\&A, 344, 409

Fabian, A. C. 1996, Science, 271, 1244

Feretti, L. 1999, in Diffuse Thermal and Relativistic Plasma in Galaxy Clusters, ed. H. Böhringer, L. Feretti, \& P. Schuecker (Garching: MPI), 3

Feretti, L., Dallacasa, D., Giovannini, G., \& Tagliani, A. 1995, A\&A, 302, 680

Feretti, L., \& Giovannini, G. 1996, in IAU Symp. 175, Extragalactic Radio Sources, ed. R. D. Ekers, C. Fanti, \& L. Padrielli (Dordrecht: Kluwer), 333

Fusco-Femiano, R., Dal Fiume, D., De Grandi, S., Feretti, L., Giovannini, G., Grandi, P., Malizia, A., Matt, G., \& Molendi, S. 2000, ApJ, 534, L7

Fusco-Femiano, R., Dal Fiume, D., Feretti, L., Giovannini, G., Grandi, P., Matt, G., Molendi, S., \& Santangelo, A. 1999, ApJ, 513, L21

Fusco-Femiano, R., Dal Fiume, D., Orlandini, M., Brunetti, G., Feretti, L., \& Giovannini, G. 2001, ApJ, 552, L97

Gaisser, T. K. 1990, Cosmic Rays and Particle Physics (Cambridge: Cambridge Univ. Press)

Gavazzi, G., \& Trinchieri, G. 1983, ApJ, 270, 410

Ginzburg, V. L. 1979, Theoretical Physics and Astrophysics (Oxford: Pergamon)

Ginzburg, V. L., \& Syrovatskii, S. I. 1965, ARA\&A, 3, 297

Giovannini, G., \& Feretti, L. 2000, NewA, 5, 335

Giovannini, G., Feretti, L., \& Stanghellini, C. 1991, A\&A, 252, 528

Giovannini, G., Feretti, L., Venturi, T., Kim, K.-T., \& Kronberg, P. P. 1993, ApJ, 406, 399

Goss, W. M., Ekers, R. D., Skellern, D. J., \& Smith, R. M. 1982, MNRAS, 198,259

Govoni, F., Enßlin, T., Feretti, L., \& Giovannini, G. 2001, A\&A, 369, 441

Hanish, R. J. 1982, A\&A, 116, 137

Jaffe, W. J. 1977, ApJ, 212, 1

Jones, T. W., Ryu, D., \& Engel, A. 1999, ApJ, 512, 105

Kaastra, J. S. 2000, in Broad Band X-Ray Spectra of Cosmic Sources, ed. K. Makishima, L. Piro, \& T. Takahashi (Nagoja: Pergamon), 741

Kaastra, J. S., Lieu, R., Mittaz, J. P. D., Bleeker, J. A. M., Mewe, R., Colafrancesco, S., \& Lockman, F. J. 1999, ApJ, 519, L119

Kang, H., Cen, R., Ostriker, J. P., \& Ryu, D. 1994, ApJ, 428, 1

Kang, H., \& Jones, T. W. 1995, ÄJ, 447, 944

Kang, H., Rachen, J., \& Biermann, P. L. 1997, MNRAS, 286, 257

Kang, H., Ryu, D., \& Jones, T. W. 1996, ApJ, 456, 422

Kim, K.-T., Kronberg, P. P., Dewdney, P. E., \& Landecker, T. L. 1990, ApJ, 355, 29

Kim, K.-T., Kronberg, P. P., Giovannini, G., \& Venturi, T. 1989, Nature, 341,720

Kulsrud, R. M., Cen, R., Ostriker, J. P., \& Ryu, D. 1997, ApJ, 480, 481

Lacey, C., \& Cole, S. 1994, MNRAS, 271, 676

Lee, M. A. 1982, J. Geophys. Res., 87, 5063

Leslie, P. R. R., \& Elsmore, B. 1961, Observatory, 81, 14

Liang, H. 1999, in Diffuse Thermal and Relativistic Plasma in Galaxy Clusters, ed. H. Böhringer, L. Feretti, \& P. Schuecker (Garching: MPI), 3

Liang, H., Hunstead, R. W., Birkinshaw, M., \& Andreani, P. 2000, ApJ, 544,686
Lieu, R., Bonamente, M., \& Mittaz, J. P. D. 1999, ApJ, 517, L91

Lieu, R., Mittaz, J. P. D., Bowyer, S., Breen, J. O., Lockman, F. J., Murphy, E. M., \& Hwang, C.-Y. 1996a, Science, 274, 1335

Lieu, R., Mittaz, J. P. D., Bowyer, S., Lockman, F. J., Hwang, C.-Y., \& Schmitt, J. H. M. M. 1996b, ApJ, 458, L5

Malkov, M. A. 1998, Phys. Rev. E, 58, 4911

Mannheim, K., \& Schlickeiser, R. 1994, A\&A, 286, 983

McClements, K. G., Dendy, R. O., Bingham, R., Kirk, J. G., \& Drury, L. O. 1997, MNRAS, 291, 241

Meyer, J.-P., Drury, L. O., \& Ellison, D. C. 1997, ApJ, 487, 182

Miniati, F. 2000, Ph.D. thesis, Univ. Minnesota

2001, Comput. Phys. Commun in press

Miniati, F., Ryu, D., Kang, H., \& Jones, T. W. 2001, ApJ, 559, 59

Miniati, F., Ryu, D., Kang, H., Jones, T. W., Cen, R., \& Ostriker, J. 2000 ApJ, 542, 608

Mittaz, J. P. D., Lieu, R., \& Lockman, F. J. 1998, ApJ, 498, L17

Mohr, J. J., Mathiesen, B., \& Evrard, A. E. 1999, ApJ, 517, 627

Moskalenko, I. V., \& Strong, A. W. 1998, ApJ, 493, 694

Mueller, D., \& Tang, K.-K. 1987, ApJ, 312, 183

Mueller, D., et al. 1995, in Proc. 25th Int. Cosmic Ray Conf. (Rome), 3, 13

Ostriker, J. P. 1993, ARA\&A, 31, 689

Ostriker, J. P., \& Cen, R. 1996, ApJ, 464, 27

Petrosian, V. 2001, ApJ, 557, 560

Quest, K. B. 1988, J. Geophys. Res., 93, 9649

Rephaeli, Y. 1977, ApJ, 212, 608

. 1979, ApJ, 227, 364

Roettiger, K., Burns, J. O., \& Locken, C. 1993, ApJ, 407, L53

Roettiger, K., Burns, J. O., \& Stone, J. M. 1999a, ApJ, 518, 603

Roettiger, K., Stone, J. M., \& Burns, J. O. 1999b, ApJ, 518, 594

Rottgering, H. J. A., Snellen, I., Miley, G., de Jong, J. P., Hanish, R. J., \& Perley, R. 1994, ApJ, 436, 654

Rottgering, H. J. A., Wieringa, M. H., Hunstead, R. W., \& Ekers, R. D. 1997, MNRAS, 290, 577

Rybicki, G. B., \& Lightman, A. P. 1979, Radiative Processes in Astrophysics (New York: Wiley)

Ryu, D., Kang, H., \& Biermann, P. L. 1998, A\&A, 335, 19

Ryu, D., Ostriker, J. P., Kang, H., \& Cen, R. 1993, ApJ, 414, 1

Sarazin, C. L. 1999, ApJ, 520, 529

Sarazin, C. L., \& Kempner, J. C. 2000, ApJ, 533, 73

Sarazin, C. L., \& Lieu, R. 1998, ApJ, 494, L177

Slee, O., Roy, A., Murgia, M., Andernach, H., \& Ehle, M. 2001, AJ, 122, 1172

Strong, A. W., \& Moskalenko, I. V. 1998, ApJ, 509, 212

Takizawa, M., \& Naito, T. 2000, ApJ, 535, 586

Tregillis, I. L. 2001, Ph.D. thesis, Univ. Minnesota

Tribble, P. C. 1993, MNRAS, 263, 31

Valtaoja, E. 1984, A\&A, 135, 141

Venturi, T., Giovannini, G., \& Feretti, L. 1990, AJ, 99, 1381

Vestrand, W. T. 1982, AJ, 87, 1266

Völk, H. J., Aharonian, F. A., \& Breitschwerdt, D. 1996, Space Sci. Rev., 75, 279

Wetherall, J. C., \& Eilek, J. A. 1999, in Diffuse Thermal and Relativistic Plasma in Galaxy Clusters, ed. H. Böhringer, L. Feretti, \& P. Schuecker (Garching: MPI), 254

White, S. D. M., Navarro, J. F., Evrard, A. E., \& Frenk, C. S. 1993, Nature, 366, 429

Willson, M. A. G. 1970, MNRAS, 151, 1 